CTP TAMU-32/00 UPR-910-T UM-TH-00-29 RUNHETC-2000-43 hep-th/0011023

November, 2000

\title{
Brane Resolution Through Transgression
}

\author{
M. Cvetič ${ }^{\dagger 1}$, H. Lü ${ }^{\star 2}$ and C.N. Pope ${ }^{\ddagger 3}$ \\ ${ }^{\dagger}$ Department of Physics and Astronomy \\ University of Pennsylvania, Philadelphia, PA 19104 \\ † Department of Physics and Astronomy, Rutgers University, Piscataway, NJ 08855 \\ ${ }^{\star}$ Department of Physics \\ University of Michigan, Ann Arbor, Michigan 48109 \\ ${ }^{\ddagger}$ Center for Theoretical Physics \\ Texas A\&M University, College Station, TX 77843 \\ ‡Institut Henri Poincaré \\ 11 rue Pierre et Marie Curie, F 75231 Paris Cedex 05
}

\begin{abstract}
$\underline{\text { ABSTRACT }}$
Modifications to the singularity structure of D3-branes that result from turning on a flux for the R-R and NS-NS 3-forms (fractional D3-branes) provide important gravity duals of four-dimensional $N=1$ super-Yang-Mills theories. We construct generalisations of these modified $p$-brane solutions in a variety of other cases, including heterotic 5-branes, dyonic strings, M2-branes, D2-branes, D4-branes and type IIA and type IIB strings, by replacing the flat transverse space with a Ricci-flat manifold $M_{n}$ that admits covariantly constant spinors, and turning on a flux built from a harmonic form in $M_{n}$, thus deforming the original solution and introducing fractional branes. The construction makes essential use of the Chern-Simons or "transgression" terms in the Bianchi-identity or equation of motion of the field strength that supports the original undeformed solution. If the harmonic form is $L^{2}$ normalisable, this can result in a deformation of the brane solution that is free of singularities, thus providing viable gravity duals of field theories in diverse dimensions that have less than maximal supersymmetry. We obtain examples of non-singular heterotic 5-branes, dyonic strings, M2-branes, type IIA strings, and D2-branes.
\end{abstract}

\footnotetext{
${ }^{1}$ Research supported in part by DOE grant DE-FG02-95ER40893 and NATO grant 976951.

${ }^{2}$ Research supported in full by DOE grant DE-FG02-95ER40899

${ }^{3}$ Research supported in part by DOE grant DE-FG03-95ER40917.
} 


\section{Contents}

\begin{tabular}{lll}
\hline & Introduction & 2
\end{tabular}

2 D3-branes on Ricci-flat Kähler 6-manifolds 5

2.1 General discussion . . . . . . . . . . . . . . . 5

2.2 D3-brane on a Ricci-flat Kähler 6-manifold $\ldots \ldots \ldots \ldots$. . . . . . 7

3 Heterotic 5-branes on Ricci-flat Kähler 4-manifolds 10

3.1 General discussion . . . . . . . . . . . . . . . . . . . . . . 10

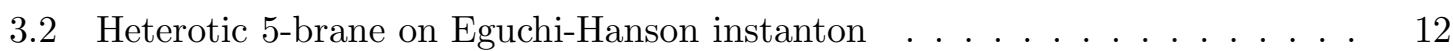

3.3 Heterotic 5 -brane on Taub-NUT instanton . . . . . . . . . . . . . 15

3.4 Heterotic 5-branes on multi Eguchi-Hanson and Taub-NUT instantons . . . 17

4 Dyonic strings on Ricci-flat Kähler 4-manifolds 19

\begin{tabular}{|lll}
5 & M2-branes on 8-manifolds & 21
\end{tabular}

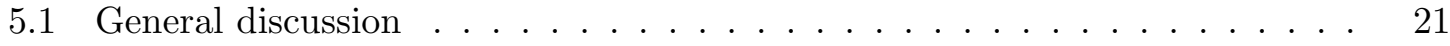

$5.2 \quad$ M2-brane on 8 -manifold of $\operatorname{Spin}(7)$ holonomy $\ldots \ldots \ldots \ldots$. . . . . . 22

6 D2-branes on 7-manifolds 25

6.1 General discussion $\ldots \ldots \ldots \ldots \ldots \ldots \ldots \ldots$

6.2 D2-brane on 7 -manifold with $G_{2}$ holonomy $\ldots \ldots \ldots \ldots \ldots$

\begin{tabular}{lll}
\hline 7 & Further examples & 28
\end{tabular}

$7.1 \quad$ Type IIA and type IIB strings on 8-manifolds . . . . . . . . . . . . . . 28

7.2 D4-branes on 5 -manifolds $\ldots \ldots \ldots \ldots \ldots \ldots \ldots$

\begin{tabular}{lll}
\hline 8 & Conclusions & 30
\end{tabular} 


\section{Introduction}

Chern-Simons type modifications, or "transgressions," in the Bianchi identities or equations of motion for field strengths are a commonplace in supergravity theories. These terms play an important role in the theory, governing both the bosonic symmetries and the supersymmetry. In the usual construction of a $p$-brane solution, one usually chooses the configuration of non-vanishing field strengths to be such that the Chern-Simons terms give no contribution in the equations of motion. In this paper, we obtain new types of brane solutions where the Chern-Simons type terms play an essential and intrinsic rôle. These solutions can be viewed as deformations of the standard brane solutions, in which addition flux is turned on, and in certain cases the deformation can have the effect of "resolving" the singularities of the original solution. Since the resolution of such BPS supergravity solutions can break additional supersymmetry, such non-singular solutions are of special interest, since they may serve as viable gravity duals of strongly-coupled Yang-Mills field theories with less than maximal supersymmetry, and thus may provide important information on these field theories, such as confinement and chiral-symmetry breaking.

Our construction applies to the general class of theory where there is an $n$-form field strength with the Bianchi identity

$$
d F_{(n)}=F_{(p)} \wedge F_{(q)}
$$

The field $F_{(n)}$ supports a standard magnetic brane solution? with a flat transverse space of dimension $n+1=p+q$. One can always replace this transverse space with any other Ricci-flat space, and provided that it admits covariantly-constant (sometimes known as "parallel") spinors, one can arrange by suitable orientation choices that the resulting brane solution will still preserve some supersymmetry. This replacement of the transverse-space metric is a key part of the construction that follows.

If the transverse space admits a suitable non-trivial harmonic $p$-form, we can take $F_{(p)}$ to be equal to this harmonic form, with the consequence that the Chern-Simons term can contribute a non-trivial flux to $F_{(n)}$. (In some cases $p=q$ and $F_{(q)}$ is the same field $F_{(p)}$, while in other cases it can be a different field.) In the cases when the harmonic forms are

\footnotetext{
${ }^{1}$ U-duality transformations can then map the solution into ones where the Chern-Simons terms play a non-trivial rôle, but these are not the type of solutions that we wish to consider in this paper, precisely because such solutions are U-duality-related to ones not involving the Chern-Simons terms.

${ }^{2}$ In some of our examples, $F_{(n)}$ itself is the Hodge dual of the "actual" field strength that appears in the theory, and so it is then field equation, rather than the Bianchi identity, that receives the Chern-Simons type correction in such a case. The field strength in the theory then carries an electric charge.
} 
normalisable, this additional flux can smooth out singularities in the solution. If on the other hand the harmonic form is non-normalisable, then we find that this leads to pathologies in the solutions. If the non-normalisablity arises due to a small-radius divergence of the harmonic function, the deformed brane solution has a (naked) singularity, whilst if there is a large-radius divergence in the norm, the solution will not have a well-defined ADM mass.

Recently, a resolution procedure has been extensively discussed, for the D3-brane solution of the type IIB theory [1, 2, 3, 4, 5], since such non-singular examples may provide important suprgravity dual solutions of four-dimensional $\mathrm{N}=1$ super-Yang-Mills theory in the infra-red. [5] The Bianchi identity for the self-dual 5 -form is given by $d F_{(5)}=-\mathrm{i} F_{(3)} \wedge \bar{F}_{(3)}$, where $F_{(3)}$ is the complex 3 -form whose real and imaginary parts are the R-R and NSNS 3-forms of the theory. The basic idea now is that one can choose any Ricci-flat sixdimensional manifold $M_{6}$ for the transverse space, and if it is additionally Kähler, it will admit covariantly-constant spinors and hence still allow a supersymmetric D3-brane solution. Then, one can look for a suitable 3 -form in $M_{6}$. It turns out that if it is harmonic, and (complex) self-dual, the type IIB supergravity equations can still be satisfied, providing the R-R and NS-NS 3-forms equal to the self-dual harmonic 3-form on $M_{6}$, thus introducing fractional branes 11]. Depending upon the detailed properties of the harmonic form, it may be that the resulting generalisation of the D3-brane solution becomes regular, thus providing a valid supergravity solution that is dual to $N=1$ four-dimensional super-Yang-Mills theory in the infra-red region.

In this paper we begin, in section 2, by reviewing the resolved solutions for the D3-brane. We also study some aspects of the specific example of the "resolved conifold" introduced in [5], which is an alternative deformation of the conifold to the one discussed previously in 1, 2, 3, 4]. In the remainder of the paper, we generalise the procedure to various other dimensions, and present a construction of new brane solutions in these cases.

In section 3 we show how one can replace the 4-dimensional space transverse to the heterotic 5-brane by any Ricci-flat space $M_{4}$, and set a $U(1)$ field chosen from the Yang-Mills sector equal to a self-dual (or anti-self-dual) harmonic 2-form in $M_{4}$. By taking $M_{4}$ to be Kähler, we have the possibility of also partially preserving the supersymmetry. We consider several examples, including the cases where $M_{4}$ is taken to be Eguchi-Hanson, Taub-NUT or the multi-centre generalisations of these metrics. Both the cases of Eguchi-Hanson and Taub-NUT admit a normalisable self-dual and anti-self-dual harmonic 2-form, respectively,

\footnotetext{
${ }^{3}$ Related aspects of branes on resolved conifolds were addressed, e.g., in [6, 7, 8], and the study of nonsingular supergravity gravity duals of $\mathrm{N}=1$ super-Yang-Mills was initiated in [9]. See also related work [10].
} 
and we find that the resulting solutions give a non-singular supersymmetric resolutions of the heterotic 5-brane. The Eguchi-Hanson metric is of further interest because it can be used to smooth out the sixteen orbifold singularities of $T^{4} / Z_{2}$, as an orbifold construction of the K3 manifold [12, 13]. Thus our solution can be viewed as the 5-brane on a K3 manifold, interpolating between a flat region in the near-orbifold limit of K3 and the curved region close to an orbifold point. It is striking that the 5-brane on the transverse K3 manifold is completely regular and does not require external source terms, whilst the 5 -brane on the transverse Euclidean space would require a 5-brane source action.

In section 4 we consider generalised dyonic string solutions in six dimensions. The construction here is somewhat similar to the heterotic 5-brane, in that the transverse space is again 4-dimensional. We again replace the transverse space by a Ricci-flat Kähler manifold $M_{4}$, and show that again we can obtain generalised solutions if there is a self-dual or anti-self-dual harmonic 2-form on $M_{4}$. Since the Eguchi-Hanson (Taub-NUT) metric has a non-trivial normalisable self-dual (anti-self-dual) harmonic 2-form we can obtain explicit non-singular resolutions of the dyonic string. Interestingly, this mechanism can also provide a resolution of repulson-type singularities for the case of tension-less dyonic strings.

In section 5, we consider generalisations of the M2-brane solution of M-theory, in which the 8-dimensional transverse space is replaced by a Ricci-flat 8-manifold $M_{8}$. There are several types of such manifolds $M_{8}$ that admit covariantly constant spinors, namely hyperKähler, with $S p(2)$ holonomy; Kähler, with $S U(4)$ holonomy; and then the exceptional case in Berger's classification [14], with $\operatorname{Spin}(7)$ holonomy. These admit 4, 2 and 1 covariantlyconstant spinors respectively, and these numbers reflect themselves in the fractions of preserved supersymmetry in the M2-brane solutions. We find that if $M_{8}$ admits a suitable self-dual (or anti-self-dual) harmonic 4-form, one can add this to the 4-form field strength, and still obtain a solution of the eleven-dimensional equations of motion. This gives generalisations of the M2-brane solution, which would, for a suitable harmonic 4-form, give rise to a non-singular resolution of the original M2-brane. We discuss an example in detail, for an $M_{8}$ manifold of Spin(7) holonomy whose Ricci-flat metric was constructed in [15, 16]. We find a normalisable harmonic 4-form, and we then use this to construct a resolved M2-brane solution that is somewhat analogous to the one we found for the heterotic 5-brane with the Eguchi-Hanson transverse metric.

In section 6 we consider generalisations of the D2-brane solution of the type IIA theory. These involve replacing the 7-dimensional flat transverse space by a Ricci-flat 7-manifold $M_{7}$. We show that if $M_{7}$ admits an harmonic 3 -form $L_{(3)}$, then one can construct generalised 
D2-brane solutions in which the 3 -form $F_{(3)}$ is taken to be proportional to $L_{(3)}$, while a term proportional to $* L_{(3)}$ is added to the 4 -form $F_{(4)}$. (Here $*$ denotes the Hodge dual in the 7-dimensional metric on $M_{7}$.) Cases that admit covariantly-constant spinors include the exceptional manifolds of $G_{2}$ holonomy occurring in the Berger classification 14. We construct an example of a generalised D2-brane, using a simple 7-manifold of $G_{2}$ holonomy. This particular example yields a linearly non-normalisable harmonic form in the asymptotic region, and the corresponding D2-brane is non-singular everywhere, but the function $H$ does not fall off fast enough asymptotically to give a well-defined ADM mass in this case.

In section 7, we discuss a few further examples of deformed brane solutions. Specifically, we construct string solutions in the type IIA and type IIB theories, and D4-branes in the type IIA theory. The type IIA strings are nothing but diagonal dimensional reductions of the M2-branes that we construct in section 5, and so they are again supported by harmonic 4-forms in the 8-dimensional Ricci-flat transverse manifold. By contrast, the mechanism for obtaining deformed string solutions in the type IIB theory is rather different, and in this case we find that they arise if the 8-manifold has an harmonic 3-form. Finally, the D4-brane solutions involve a Ricci-flat 5-dimensional transverse space, with an harmonic 2-form.

In section 8 we summarise the results, and comment on possible generalisations and further studies in connection with field-theory duals.

\section{D3-branes on Ricci-flat Kähler 6-manifolds}

\subsection{General discussion}

We begin with a brief review of the deformed D3-brane solutions, which have been discussed

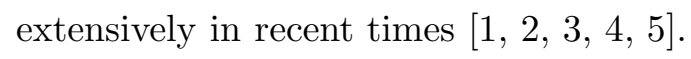

The bosonic sector of ten-dimensional type IIB supergravity comprises the metric, a self-dual 5-form field strength, a scalar, an axion, an R-R 3-form and an NS-NS 3-form field strength. There is no simple covariant Lagrangian for type IIB supergravity, on account of the self-duality constraint for the 5-form. However, one can write a Lagrangian in which the 5 -form is unconstrained, which must then be accompanied by a self-duality condition which is imposed by hand at the level of the equations of motion 17]. This type IIB Lagrangian is

$$
\begin{aligned}
\mathcal{L}_{10}^{\mathrm{IIB}}= & \hat{R} \hat{*} \mathbb{1}-\frac{1}{2} \hat{*} d \phi \wedge d \phi-\frac{1}{2} e^{2 \phi} \hat{*} d \chi \wedge d \chi-\frac{1}{4} \hat{*} F_{(5)} \wedge F_{(5)} \\
& -\frac{1}{2} e^{-\phi} \hat{*} F_{(3)}^{\mathrm{NS}} \wedge F_{(3)}^{\mathrm{NS}}-\frac{1}{2} e^{\phi} \hat{*} F_{(3)}^{\mathrm{RR}} \wedge F_{(3)}^{\mathrm{RR}}-\frac{1}{2} B_{(4)} \wedge d A_{(2)}^{\mathrm{RR}} \wedge d A_{(2)}^{\mathrm{NS}},
\end{aligned}
$$

where $F_{(3)}^{\mathrm{NS}}=d A_{(2)}^{\mathrm{NS}}, F_{(3)}^{\mathrm{RR}}=d A_{(2)}^{\mathrm{RR}}-\chi d A_{(2)}^{\mathrm{NS}}, F_{(5)}=d B_{(4)}-\frac{1}{2} A_{(2)}^{\mathrm{RR}} \wedge d A_{(2)}^{\mathrm{NS}}+\frac{1}{2} A_{(2)}^{\mathrm{NS}} \wedge d A_{(2)}^{\mathrm{RR}}$. Note 
that $A_{(2)}^{\mathrm{RR}}$ and $A_{(2)}^{\mathrm{NS}}$ are the R-R and NS-NS 2-form potentials respectively. Our convention here, and throughout the paper, is that the metric in the theory that we are considering will be denoted by $d \hat{s}^{2}$, and likewise all quantities associated with it, such as the Ricci tensor $\hat{R}$, will carry hats. In particular, $\hat{*}$ in the present case denotes the ten-dimensional Hodge dual.

One can then make the following "deformed" D3-brane Ansatz,

$$
\begin{aligned}
d \hat{s}_{10}^{2} & =H^{-1 / 2} d x^{\mu} d x^{\nu} \eta_{\mu \nu}+H^{1 / 2} d s_{6}^{2}, \\
F_{(5)} & =d^{4} x \wedge d H^{-1}+\hat{*} d H, \quad F_{3} \equiv F_{(3)}^{\mathrm{RR}}+\mathrm{i} F_{(3)}^{\mathrm{NS}}=m L_{(3)},
\end{aligned}
$$

where $d s_{6}^{2}$ is any six-dimensional Ricci-flat Kähler metric that admits a non-trivial complex harmonic self-dual 3-form $L_{(3)}=\mathrm{i} * L_{(3)}$, and $*$ is the Hodge dual with respect to $d s_{6}^{2}$. Again, our notation here and throughout the paper is that the unhatted metric always denotes the one in the space transverse to the brane world-volume, and $*$ denotes the Hodge dual with respect to this metric.

It is straightforward to verify the following:

$$
\begin{aligned}
d \hat{*} F_{(3)}=\mathrm{i} F_{(5)} \wedge F_{(3)} & \Rightarrow \mathrm{i} * L_{(3)}=L_{(3)}, \quad d L_{(3)}=0, \\
(\chi, \phi) \text { equations } & \Rightarrow \mathrm{i} * L_{(3)}=L_{(3)}, \\
d F_{(5)}=-\mathrm{i} F_{(3)} \wedge \bar{F}_{(3)} & \Rightarrow \square H=-\frac{1}{12} m^{2}\left|L_{(3)}\right|^{2}, \\
\text { Einstein equation } & \Rightarrow \square H=-\frac{1}{12} m^{2}\left|L_{(3)}\right|^{2},
\end{aligned}
$$

where

$$
\left|L_{(3)}\right|^{2} \equiv L_{m n p} \bar{L}^{m n p}
$$

and $\square$ denotes the scalar Laplacian calculated in the six-dimensional transverse-space with metric $d s_{6}^{2}$. Thus all the equations of motion are satisfied provided that

$$
\square H=-\frac{1}{12} m^{2}\left|L_{(3)}\right|^{2} .
$$

Note that there is a correlation between the sign in the duality equation $\mathrm{i} * L_{(3)}=L_{(3)}$, and the sign of the Chern-Simons or transgression term in the type IIB theory.

We see that the complex self-dual harmonic 3 -form $L_{(3)}$ acts as a source for the function $H$. In Euclidean 6-space, there is always a simple harmonic form 3-form for which $L_{(3)} \wedge \bar{L}_{(3)}$ becomes the volume form. This is not especially interesting since it contributes a term $-m^{2} r^{2}$ to the function $H$, which implies that the solution contains a naked singularity. More interesting solutions can be obtained by taking the transverse space to be some nontrivial complete non-compact Ricci-flat 6-manifold. An example that has been much studied 
is the "deformed conifold" that was introduced in [18], and studied further in [19, 20]. The deformed D3-brane solution using this metric was constructed in [2]. In section 2.2, we shall study some aspects of another deformed D3-brane solution that has recently been discussed, using a different complete Ricci-flat 6-manifold [5].

\subsection{D3-brane on a Ricci-flat Kähler 6-manifold}

The transverse 6-metric that we shall consider here is the one discussed recently in [5], where it was used to obtain a deformed D3-brane solution with NS-NS and R-R flux. It is a metric of cohomogeneity one, whose level surfaces are the 5-dimensional manifold of the $U(1)$ bundle over $S^{2} \times S^{2}$, where the $U(1)$ fibre has winding number 1 over each 2 -sphere. The metric can be written as

$$
d s_{6}^{2}=h^{2} d r^{2}+\alpha^{2} \sigma^{2}+\beta^{2} d \Omega_{2}^{2}+\gamma^{2} d \widetilde{\Omega}_{2}^{2},
$$

where $h, \alpha, \beta$ and $\gamma$ are functions only of $r$, and

$$
\begin{aligned}
& d \Omega_{2}^{2}=d \theta^{2}+\sin ^{2} \theta d \phi^{2}, \quad d \widetilde{\Omega}_{2}^{2}=d \tilde{\theta}^{2}+\sin ^{2} \tilde{\theta} d \tilde{\phi}^{2} \\
& \sigma=d \psi+\cos \theta d \phi+\cos \tilde{\theta} d \tilde{\phi}
\end{aligned}
$$

Choosing $e^{0}=h d r, e^{1}=\beta d \theta, e^{2}=\beta \sin \theta d \phi, e^{3}=\gamma d \tilde{\theta}, e^{4}=\gamma \sin \tilde{\theta} d \tilde{\phi}, e^{5}=\alpha \sigma$ for the orthonormal frame, the tangent-space components of the Ricci tensor are given by

$$
\begin{aligned}
& R_{00}=-\frac{1}{h \alpha}\left(\frac{\alpha^{\prime}}{h}\right)^{\prime}-\frac{2}{h \beta}\left(\frac{\beta^{\prime}}{h}\right)^{\prime}-\frac{2}{h \gamma}\left(\frac{\gamma^{\prime}}{h}\right)^{\prime}, \\
& R_{11}=R_{22}=-\frac{1}{h \beta}\left(\frac{\beta^{\prime}}{h}\right)^{\prime}-\frac{\beta^{\prime 2}}{h^{2} \beta^{2}}-\frac{2 \beta^{\prime} \gamma^{\prime}}{h^{2} \beta \gamma}-\frac{\alpha^{\prime} \beta^{\prime}}{h^{2} \alpha \beta}+\frac{1}{\beta^{2}}-\frac{\alpha^{2}}{2 \beta^{4}}, \\
& R_{33}=R_{44}=-\frac{1}{h \gamma}\left(\frac{\gamma^{\prime}}{h}\right)^{\prime}-\frac{\gamma^{\prime 2}}{h^{2} \gamma^{2}}-\frac{2 \beta^{\prime} \gamma^{\prime}}{h^{2} \beta \gamma}-\frac{\alpha^{\prime} \gamma^{\prime}}{h^{2} \alpha \gamma}+\frac{1}{\gamma^{2}}-\frac{\alpha^{2}}{2 \gamma^{4}}, \\
& R_{55}=-\frac{1}{h \alpha}\left(\frac{\alpha^{\prime}}{h}\right)^{\prime}+\frac{\alpha^{2}}{2 \beta^{4}}+\frac{\alpha^{2}}{2 \gamma^{4}}-\frac{2 \alpha^{\prime} \beta^{\prime}}{h^{2} \alpha \beta}-\frac{2 \alpha^{\prime} \gamma^{\prime}}{h^{2} \alpha \gamma} .
\end{aligned}
$$

It is then easily verified that the following gives a Ricci-flat metric [5]:

$$
h^{2}=\frac{r^{2}+6 a^{2}}{r^{2}+9 a^{2}}, \quad \alpha^{2}=\frac{1}{9}\left(\frac{r^{2}+9 a^{2}}{r^{2}+6 a^{2}}\right) r^{2}, \quad \beta^{2}=\frac{1}{6} r^{2}, \quad \gamma^{2}=\frac{1}{6}\left(r^{2}+6 a^{2}\right),
$$

where $a$ is a constant. The radial coordinate runs from $r=0$ to $r=\infty$. Near $r=0$, the metric smoothly approaches flat $R^{4}$ times a 2-sphere of radius $a$, while at large $r$ the metric describes the cone with level surfaces that are the $U(1)$ bundle over $S^{2} \times S^{2}$. If the parameter $a$ is set to zero, the cone metric becomes singular at $r=0$, and the manifold degenerates to the conifold described in detail in [18]. The construction of the homogeneous metrics on the $U(1)$ bundle over $S^{2} \times S^{2}$ that is used here was given in [21]. 
Substituting (2.9) back into the expressions for the curvature, one finds that the curvature 2-forms $\Theta_{a b}=\frac{1}{2} R_{a b c d} e^{c} \wedge e^{d}$ are given by

$$
\begin{aligned}
& \Theta_{01}=A\left(e^{0} \wedge e^{1}+e^{2} \wedge e^{5}\right), \quad \Theta_{02}=A\left(e^{0} \wedge e^{2}-e^{1} \wedge e^{5}\right) \\
& \Theta_{03}=-B\left(e^{0} \wedge e^{3}+e^{4} \wedge e^{5}\right), \quad \Theta_{04}=-B\left(e^{0} \wedge e^{4}-e^{3} \wedge e^{5}\right), \\
& \Theta_{05}=\frac{72 a^{4}}{\left(r^{2}+6 a^{2}\right)^{3}}\left(e^{0} \wedge e^{5}+e^{3} \wedge e^{4}\right)-\frac{6 a^{2}}{\left(r^{2}+6 a^{2}\right)^{2}}\left(e^{1} \wedge e^{2}-e^{3} \wedge e^{4}\right), \\
& \Theta_{12}=\frac{r^{2}}{r^{2}+6 a^{2}}\left(e^{1} \wedge e^{2}-e^{3} \wedge e^{4}\right)-\frac{6 a^{2}}{\left(r^{2}+6 a^{2}\right)^{2}}\left(e^{0} \wedge e^{5}+e^{3} \wedge e^{4}\right) \\
& \Theta_{13}=-C\left(e^{1} \wedge e^{3}+e^{2} \wedge e^{4}\right), \quad \Theta_{14}=-C\left(e^{1} \wedge e^{4}-e^{2} \wedge e^{3}\right) \\
& \Theta_{15}=A\left(e^{1} \wedge e^{5}-e^{0} \wedge e^{2}\right), \quad \Theta_{23}=-C\left(e^{2} \wedge e^{3}-e^{1} \wedge e^{4}\right) \\
& \Theta_{24}=-C\left(e^{2} \wedge e^{4}+e^{1} \wedge e^{3}\right), \quad \Theta_{25}=A\left(e^{2} \wedge e^{5}+e^{0} \wedge e^{1}\right) \\
& \Theta_{35}=-B\left(e^{3} \wedge e^{5}-e^{0} \wedge e^{4}\right), \quad \Theta_{45}=-B\left(e^{4} \wedge e^{5}+e^{0} \wedge e^{3}\right)
\end{aligned}
$$

where we have defined

$$
A \equiv \frac{3 a^{2}}{\left(r^{2}+6 a^{2}\right)^{2}}, \quad B \equiv \frac{3 a^{2}\left(r^{2}+18 a^{2}\right)}{\left(r^{2}+6 a^{2}\right)^{3}}, \quad C \equiv \frac{r^{2}+9 a^{2}}{\left(r^{2}+6 a^{2}\right)^{2}} .
$$

The metric is Kähler, with Kähler form given by

$$
J=e^{1} \wedge e^{2}+e^{3} \wedge e^{4}-e^{0} \wedge e^{5} .
$$

Since the integrability condition for the existence of a covariantly-constant spinor is $R_{a b c d} \Gamma^{c d} \eta=0$, we can immediately deduce from the expressions for the curvature 2-forms that $\eta$ must satisfy

$$
\Gamma_{12} \eta=\Gamma_{34} \eta=-\Gamma_{05} \eta
$$

It is then straightforward to substitute back into the covariant-constancy equation itself, $D_{a} \eta=0$, to deduce that $\eta$ is given by

$$
\eta=e^{-\frac{1}{2} \psi \Gamma_{12}} \eta_{(0)}
$$

where $\eta_{(0)}$ is a constant spinor that satisfies the same conditions 2.13). There are two such independent solutions.

As in [5], one may note that the following is an harmonic 3-form:

$$
\omega_{(3)}=\sigma \wedge \Omega_{(2)}-\sigma \wedge \widetilde{\Omega}_{(2)},
$$

where $\Omega_{(2)} \equiv \sin \theta \wedge d \phi$ and $\widetilde{\Omega}_{(2)} \equiv \sin \tilde{\theta} \wedge d \tilde{\phi}$ are the volume forms on the two unit 2-spheres. The Hodge dual of $\omega_{(3)}$ is given by

$$
* \omega_{(3)}=\frac{h \gamma^{2}}{\alpha \beta^{2}} d r \wedge \widetilde{\Omega}_{(2)}-\frac{h \beta^{2}}{\alpha \gamma^{2}} d r \wedge \Omega_{(2)} .
$$


Thus the closure and co-closure of $\omega_{(3)}$ is manifest.

It is clear that from $\omega_{(3)}$ we can construct the complex self-dual harmonic 3-form

$$
L_{(3)}=\omega_{(3)}+\mathrm{i} * \omega_{(3)},
$$

satisfying $L_{(3)}=\mathrm{i} * L_{(3)}$. It is then easy to see that

$$
\left|L_{(3)}\right|^{2}=\frac{7776\left(18 a^{4}+6 a^{2} r^{2}+r^{4}\right)}{r^{6}\left(r^{2}+6 a^{2}\right)\left(r^{2}+9 a^{2}\right)} .
$$

This harmonic 3-form is not normalisable, owing to the strength of its divergence as $r \rightarrow 0$, and so we can expect that the deformed D3-brane will have a singularity for small $r$. Furthermore, its fall-off at large $r$ is insufficient to give normalisability there; there will be a logarithmic divergence.

If we assume the function $H$ depends only on $r$, then (2.5) becomes

$$
\left(r^{3}\left(r^{2}+9 a^{2}\right) H^{\prime}\right)^{\prime}=-\frac{648 m^{2}\left(18 a^{4}+6 a^{2} r^{2}+r^{4}\right)}{r^{3}\left(r^{2}+9 a^{2}\right)} .
$$

The first integration of this equation gives

$$
r^{3}\left(r^{2}+9 a^{2}\right) H^{\prime}=-81 b+\frac{648 a^{2} m^{2}}{r^{2}}-288 m^{2} \log r-180 m^{2} \log \left(r^{2}+9 a^{2}\right),
$$

and the second gives

$$
\begin{aligned}
H= & 1+\frac{3 b-4 m^{2}+24 m^{2} \log (3 a)}{3 a^{4}} \log r+\frac{4 m^{2}-3 b}{6 a^{4}} \log \left(r^{2}+9 a^{2}\right) \\
& +\frac{m^{2}}{9 a^{4}}\left(4 \log r-5 \log \left(r^{2}+9 a^{2}\right)\right)\left(4 \log r+\log \left(r^{2}+9 a^{2}\right)\right) \\
& +\frac{9 b+24 m^{2}+32 m^{2} \log r+20 m^{2} \log \left(r^{2}+9 a^{2}\right)}{2 a^{2} r^{2}}-\frac{18 m^{2}}{r^{4}}-\frac{2 m^{2}}{a^{4}} \operatorname{Li}_{2}\left(-\frac{r^{2}}{9 a^{2}}\right),
\end{aligned}
$$

where $\operatorname{Li}_{2}(x)=\int_{x}^{0} d t \log (1-t) / t$ is the dilogarithm function, and $b$ is a constant of integration. (This is the explicit form of the solution whose general structure was discussed in [5.) The leading-order behaviour for $H$ is given by

$$
\begin{array}{ll}
r>>a: & H=1+\frac{81\left(b+2 m^{2}\right)}{4 r^{4}}+\frac{162 m^{2} \log r}{r^{4}}+\cdots, \\
r<<a: & H=1-\frac{18 m^{2}}{r^{4}}+\frac{9 b+24 m^{2}+40 m^{2} \log (3 a)+32 m^{2} \log r}{2 a^{2} r^{2}}+\cdots .
\end{array}
$$

Thus the solution has a repulson-type singularity [5]. Furthermore the logarithmic behaviour of $H$ at large $r$ implies that the metric does not have a well-defined ADM mass. These behaviours are a direct consequences of the non-normalisability of $L_{(3)}$. Namely, since $L_{(3)}$ is not normalisable for small $r$, the solution is singular near the origin; and since it is not normalisable at large $r, H$ will fall off too slowly to have a well-defined ADM mass. 
As mentioned earlier, it is also possible to consider a different Ricci-flat 6-metric, namely the one discussed in the deformed D3-brane in [2]. In this case the 6-metric is quite distinct from the metric of [5] that we have been studying here. They are both complete, but the Ricci-flat 6-metric used in [2] has a minimal $S^{3}$ in the centre, whilst the Ricci-flat metric in [5] has a minimal $S^{2}$ in the centre. In the D3-brane solution using this "deformed conifold" that was constructed in [2], the harmonic 3-form $L_{(3)}$ is normalisable at small $r$, but logrithmically non-normalisable for large $r$. As a consequence, the function $H$ has no singularity at small $r$, but has the same logrithmic behavior at large $r$ as in the solution of [5].

The two covariantly-constant spinors, given by (2.13) and (2.14), would give rise to Killing spinors of the D3-brane solution if we merely replaced the flat transverse 6 -space of a standard D3-brane solution by the Ricci-flat manifold with the metric under discussion here. Once one turns on the NS-NS and R-R 3-form flux, by allowing the parameter $m$ to be non-zero, it is necessary to check the additional conditions that now arise. A discussion of supersymmetry in such deformed D3-brane solutions was given in [3, 使. In particular, from the results in [3] it is necessary for supersymmetry that the 3 -form $L_{(3)}$ be purely of type $(2,1)$, with no admixture of $(1,2),(0,3)$ or $(3,0)$ terms. Using the Kähler form $(2.12)$, we can verify that the complex 3 -form $L_{(3)}$ defined in (2.17) indeed has no purely holomorphic or antiholomorphic parts, of type $(0,3)$ or $(3,0)$. However, it does have terms of type $(1,2)$ as well as $(2,1)$, and so based on the results in [3], it would seem that the solution will not be supersymmetric.

\section{Heterotic 5-branes on Ricci-flat Kähler 4-manifolds}

\subsection{General discussion}

The bosonic sector of the ten-dimensional heterotic supergravity consists of the metric, a dilaton, a 2-form potential $A_{(2)}$ and the Yang-Mills fields of $E_{8} \times E_{8}$ or $S O(32)$. The Lagrangian is given by

$$
\mathcal{L}_{\text {het }}=\hat{R} \hat{*} \mathbb{1}-\frac{1}{2} \hat{*} d \phi \wedge d \phi-\frac{1}{2} e^{-\phi} \hat{*} F_{3} \wedge F_{(3)}-\frac{1}{2} e^{-\frac{1}{2} \phi} \hat{*} F_{(2)}^{i} \wedge F_{(2)}^{i},
$$

where

$$
\begin{aligned}
& F_{(3)}=d A_{(2)}+\frac{1}{2} A_{(1)}^{i} \wedge d A_{(1)}^{i}+\frac{1}{6} f_{i j k} A_{(1)}^{i} \wedge A_{(1)}^{j} \wedge A_{(1)}^{k}, \\
& F_{(2)}^{i}=d A_{(1)}^{i}+\frac{1}{2} f^{i}{ }_{j k} A_{(1)}^{j} \wedge A_{(1)}^{k} .
\end{aligned}
$$


The Bianchi identity for $F_{(3)}$ is given by

$$
d F_{(3)}=\frac{1}{2} F_{(2)}^{i} \wedge F_{(2)}^{i} .
$$

This implies that the magnetic 5-brane charge can be supplied by a Yang-Mills instanton in the 4-space transverse to the heterotic 5-brane. Such a solution was constructed in [22], where it was shown that the singularity of the standard 5-brane is smoothed out by the instanton configuration.

Here, we show that the heterotic 5-brane admits a quite different kind of deformation, supported by an Abelian $U(1)$ field, again giving a regular solution, provided that the transverse 4 -space admits a non-trivial self-dual harmonic 2 -form. To do this, we make the following 5-brane Ansatz,

$$
\begin{aligned}
d \hat{s}_{10}^{2} & =H^{-1 / 4} d x^{\mu} d x^{\mu} \eta_{\mu \nu}+H^{3 / 4} d s_{4}^{2}, \\
e^{-\phi} \hat{*} F_{(3)} & =d^{6} x \wedge d H^{-1}, \quad \phi=\frac{1}{2} \log H, \quad F_{(2)}=m L_{(2)},
\end{aligned}
$$

where $L_{(2)}$ is an harmonic 2-form in the Ricci-flat transverse metric $d s_{4}^{2}$. Note that here we only turn on one of the Yang-Mills gauge fields, which we write as $F_{(2)}$. It is straightforward to verify that the above Ansatz satisfies all the equations of motion, provided that $L_{(2)}$ is a self-dual harmonic 2 -form, $\left(* L_{(2)}=L_{(2)}, d L_{(2)}=0\right)$ and that

$$
\square H=-\frac{1}{4} m^{2} L_{(2)}^{2} \text {. }
$$

Note that the sign in the duality relation $* L_{(2)}=+L_{(2)}$ is correlated with the sign of the Chern-Simons type terms in the expression for $F_{(3)}$ in (3.2), and hence in (3.3) too. It also depends, of course, on our orientation conventions when taking the Hodge dual. Consequently, we can perfectly well also obtain a solution of the above type in a case where we instead have an anti-self-dual harmonic 2 -form in the transverse metric $d s_{4}^{2}$, by making the appropriate orientation change. One must be careful, however, when checking the supersymmetry of the solution, since reversing the orientation of the transverse space can make the difference between whether or not covariantly-constant spinors exist that have the required chirality for obtaining supersymmetry in the generalised 5-brane solution.

Since $\phi$ and $F_{(3)}$ in the new solutions (3.4) have the same functional dependence on $H$ as they do in the standard heterotic 5-brane solution, it follows that the gravitino and dilatino transformation rules will imply very similar conditions for preserved supersymmetry to those in the standard solution where $d s_{4}^{2}$ is flat. Thus we have

$$
\begin{aligned}
\delta \psi_{M} & =\hat{D}_{M} \epsilon-\frac{1}{96} e^{-\frac{1}{2} \phi} F_{N P Q}\left(\Gamma_{M}^{N P Q}-\delta_{M}^{N} \Gamma^{P Q}\right) \epsilon=0, \\
\delta \lambda & =\partial_{M} \phi \Gamma^{M} \epsilon-\frac{1}{12} e^{-\frac{1}{2} \phi} F_{M N P} \Gamma^{M N P} \epsilon=0,
\end{aligned}
$$


which implies first of all the usual condition

$$
\frac{1}{4 !} \epsilon_{a b c d} \Gamma^{a b c d} \epsilon+\epsilon=0
$$

where the indices $a, b, \ldots$ range over the 4-dimensional transverse space. In addition, the gravitino transformation rule now requires that after decomposing $\epsilon$ as the product of a spinor in the six-dimensional brane world-volume and a spinor $\eta$ in the four-dimensional transverse space, $\eta$ must be covariantly constant in the metric $d s_{4}^{2}$. This implies that we must take the Ricci-flat metric $d s_{4}^{2}$ to be Kähler, and that the 4-manifold must be oriented appropriately. Finally, the gaugino transformation rules

$$
\delta \chi^{i}=F_{M N}^{i} \Gamma^{M N} \epsilon
$$

imply that $\eta$ must also satisfy

$$
L_{a b} \Gamma^{a b} \eta=0
$$

in order to have supersymmetry.

\subsection{Heterotic 5-brane on Eguchi-Hanson instanton}

Let us consider the case where the Ricci-flat transverse 4-metric $d s_{4}^{2}$ is the Eguchi-Hanson solution [23],

$$
\begin{aligned}
d s_{4}^{2} & =W^{-1} d r^{2}+\frac{1}{4} r^{2} W(d \psi+\cos \theta d \phi)^{2}+\frac{1}{4} r^{2} d \Omega_{2}^{2}, \\
W & =1-\frac{a^{4}}{r^{4}}
\end{aligned}
$$

where $d \Omega_{2}^{2}=d \theta^{2}+\sin ^{2} \theta d \phi^{2}$. The radial coordinate $r$ lies in the range $a \leq r \leq \infty$, and $\psi$ has period $2 \pi$. The metric is asymptotically locally Euclidean (ALE), with the periodicity condition on $\psi$ implying that that the level surfaces at constant $r$ are $R P^{3}=S^{3} / Z_{2}$. It is Kähler, with self-dual curvature. The Kähler form, which is anti-self-dual in these conventions, is given by

$$
J=\frac{1}{2} r d r \wedge(d \psi+\cos \theta d \phi)-\frac{1}{4} r^{2} \Omega_{(2)}=e^{0} \wedge e^{3}-e^{1} \wedge e^{2},
$$

where $\Omega \equiv \sin \theta d \theta \wedge d \phi$ is the volume-form of the unit 2-sphere metric $d \Omega_{2}^{2}$, and we define the orthonormal basis

$$
e^{0}=W^{-1 / 2} d r, \quad e^{1}=\frac{1}{2} r d \theta, \quad e^{2}=\frac{1}{2} r \sin \theta d \phi, \quad e^{3}=\frac{1}{2} r W^{1 / 2}(d \psi+\cos \theta d \phi) .
$$


The curvature 2-forms are given by

$$
\begin{aligned}
& \Theta_{01}=\Theta_{23}=-\frac{2 a^{4}}{r^{6}}\left(e^{0} \wedge e^{1}+e^{2} \wedge e^{3}\right), \\
& \Theta_{02}=\Theta_{31}=-\frac{2 a^{4}}{r^{6}}\left(e^{0} \wedge e^{2}+e^{3} \wedge e^{1}\right), \\
& \Theta_{03}=\Theta_{12}=\frac{4 a^{4}}{r^{6}}\left(e^{0} \wedge e^{3}+e^{1} \wedge e^{2}\right)
\end{aligned}
$$

From the integrability condition $R_{a b c d} \Gamma^{c d} \eta=0$ for covariantly-constant spinors it follows that there are two, which satisfy the projection condition

$$
\left(\Gamma_{03}+\Gamma_{12}\right) \eta=0
$$

The metric also admits a self-dual harmonic 2-form, given by

$$
L_{(2)}=r^{-3} d r \wedge(d \psi+\cos \theta d \phi)+\frac{1}{2} r^{-2} \Omega_{2}=\frac{2}{r^{4}}\left(e^{0} \wedge e^{3} \wedge e^{1} \wedge e^{2}\right) .
$$

The square of $L_{(2)}$ is given by

$$
L_{(2)}^{2}=\frac{16}{r^{8}}
$$

and so this harmonic 2-form is normalisable.

Making the assumption that $H$ depends only on $r$, we now find that (3.5) becomes

$$
\left(r^{3} W H^{\prime}\right)=-\frac{4 m^{2}}{r^{5}}
$$

The solution for $H$ is given by

$$
H=1+\frac{m^{2}+a^{4} b}{4 a^{6}} \log \left(\frac{r^{2}-a^{2}}{r^{2}+a^{2}}\right)+\frac{m^{2}}{2 a^{4} r^{2}} .
$$

where $b$ in an arbitrary integration constant. (We have chosen the second (additive) constant of integration to be 1 for convenience.) Since the coordinate $r$ runs from $a$ to infinity, it follows that in general there is a naked singularity when $r$ is close to $a$. However, we can choose the constant $b=-m^{2} / a^{4}$ such that the logarithmic term cancels, giving

$$
H=1+\frac{m^{2}}{2 a^{4} r^{2}} .
$$

Note that in the region where $r>>a$, the Eguchi-Hanson metric is asymptotically locally Euclidean, and $H$ in (3.18) or (3.19) has the usual $1 / r^{2}$ power-law fall-off. When $b=$ $-m^{2} / a^{4}$, the 5 -brane solution is completely non-singular.

The supersymmetry of the solution is easily determined. From the general discussion in section 3.1, and the condition (3.14) for covariantly-constant spinors in the Eguchi-Hanson metric, we see that the gravitino and dilatino transformation rules imply that the solution 
will preserve one half of the original supersymmetry. (Note that (3.14) just amounts to a chirality condition on $\eta$, which is the same as the condition for the usual heterotic D5brane.) Furthermore since the harmonic 2-form $L_{(2)}$ in our new solution is self-dual, given by (3.15), it immediately follows from (3.14) that the condition (3.9) following from the gaugino transformation rule is satisfied. Thus the new solution preserves one half of the original supersymmetry.

Another comment is in order. After a dimensional reduction (along $\{\theta, \phi, \psi\}$ direction), the above smooth solution can be interpreted as a BPS domain wall solution in $\mathrm{D}=7$ gauged supergravity, preserving $1 / 4$ of the original supersymmetry. It is therefore expected that the equations of motion for this configuration can be written as a coupled system of firstorder differential equations for the scalar fields and the conformal factor of the conformally flat space-time metric, which are governed by the specific form of the superpotential of the scalar fields. (These equations were first discussed in the context of four-dimensional BPS supergravity domain walls in [24]; for a review see [25].) Note that the derivation of the explicit form of the superpotential for the case in consideration would yield information on the dual six-dimensional field theory, but we relegate this derivation (as well as those of all the subsequent domain-wall examples in this paper) to further study [26].

Having obtained the supersymmetric smooth heterotic 5-brane on the Eguchi-Hanson metric, it is of interest to study the spectrum of a minimally-coupled scalar in this gravitational background, since this provides an information on the ("glue-ball") spectrum in the infra-red regime of the dual $\mathrm{N}=2$ six-dimensional field theory (see, for example, 27] and references therein). The equation is of the form

$$
\frac{1}{\sqrt{\hat{g}}} \partial_{M}\left(\sqrt{\hat{g}} \hat{g}^{M N} \partial_{N} \chi\right)=0
$$

In a suitable decoupling limit, the 1 in the function $H$ in (3.19) can be dropped. Making an Ansatz for $\chi$ with $\chi=e^{\mathrm{i} p \cdot x} H^{1 / 2} W^{-1 / 4} \psi(r)$, and performing a coordinate transformation $r^{4}=a^{4} /(\tanh (2 z)-1)$, where $z$ runs from 0 to infinity, the wave equation becomes

$$
\left(-\partial_{z}^{2}-V\right) \psi=\frac{m^{2} p^{2}}{2 a^{2}} \psi
$$

where the Schrödinger potential $V$ is given by

$$
V=\frac{\cosh (4 z)-3}{2 \sinh ^{2}(2 z)} .
$$

The potential approaches $-1 /\left(4 z^{2}\right)$ as $z \rightarrow 0$, whilst it approaches a positive constant, 1 , for large $z$. In spite of the attractive potential as $z \rightarrow 0$, it turns out that the boundary 
conditions eliminate the non-positive energy bound-states and the spectrum turns out to be continuous, with a mass gap $2 a^{2} / \mathrm{m}^{2}$.

The Eguchi-Hanson metric is particular interesting because it can be used to smooth out the sixteen orbifold singularities of $T^{4} / Z_{2}$, as an orbifold construction of the K3 manifold 12, 13. Thus our solution can be viewed as the 5-brane on K3 manifold, interpolating between a flat region in the near-orbifold limit of $\mathrm{K} 3$ and the curved region close to an orbifold point. It is striking that the 5-brane on the transverse K3 manifold is completely regular and does not require external source term, whilst the 5-brane on the transverse Euclidean space would require 5-brane action source. It is worth noting that the number of orbifold singularities of $T^{4} / Z_{2}$ is precisely the same as the number of Cartan generators of the heterotic string theories.

Note that if we take the limit where the Eguchi-Hanson scale-size $a$ tends to zero, so that $d s_{4}^{2}$ becomes locally Euclidean 4 -space, the self-dual $L_{(2)}$ will not contribute any flux. Making convenient choices for the integration constants, $H$ is then given by

$$
H=1+\frac{Q}{r^{2}}-\frac{m^{2}}{3 r^{6}} \text {. }
$$

Thus in this flat-space limiting case of the Eguchi-Hanson metric, the inclusion of the $L_{(2)}$ term now leads to a naked singularity that can no longer be removed.

We could instead have chosen the harmonic 2-form $L_{(2)}$ to be the Kähler form (3.11), which is anti-self-dual. This is not normalisable, and in fact if we take $L_{(2)}=J$ we shall have $L_{(2)}^{2}=4$. As we discussed previously, we can still use this anti-self-dual harmonic 2-form to construct a generalised 5-brane solution, provided that we first reverse the orientation of the Eguchi-Hanson manifold so that it becomes self-dual. Substituting into (3.5), we then find that $H$ is given by

$$
H=1-\frac{1}{8} m^{2} r^{2}+\frac{\left(a^{4} m^{2}-4 b\right)}{16 a^{2}} \log \left(\frac{r^{2}+a^{2}}{r^{2}-a^{2}}\right),
$$

implying an unavoidable singularity. Furthermore, it is now evident from the criterion (3.9) for supersymmetry, which comes from the gaugino transformation rule, that the anti-selfduality of $L_{(2)}$ will conflict with (3.14), and so in this solution there would be no supersymmetry.

\subsection{Heterotic 5-brane on Taub-NUT instanton}

Another example of a Ricci-flat Kähler 4-metric is the Taub-NUT instanton [28],

$$
d s_{4}^{2}=\left(\frac{r+a}{r-a}\right) d r^{2}+4 a^{2}\left(\frac{r-a}{r+a}\right)(d \psi+\cos \theta d \phi)^{2}+\left(r^{2}-a^{2}\right)\left(d \theta^{2}+\sin ^{2} \theta d \phi^{2}\right),
$$


where the radial coordinate runs from $r=a$ to $r=\infty$, and $\psi$ has period $4 \pi$. Topologically, the Taub-NUT manifold is $\mathbb{R}^{4}$, but although the metric at large $r$ is asymptotically flat, it approaches the cylinder $\mathbb{R}^{3} \times S^{1}$ rather than Euclidean space. In the obvious orthonormal frame

$$
\begin{aligned}
e^{0} & =\left(\frac{r-a}{r+a}\right)^{-1 / 2} d r, \quad e^{1}=\left(r^{2}-a^{2}\right)^{1 / 2} d \theta, \quad e^{2}=\left(r^{2}-a^{2}\right)^{1 / 2} \sin \theta d \phi, \\
e^{3} & =2 a\left(\frac{r-a}{r+a}\right)^{1 / 2}(d \psi+\cos \theta d \phi),
\end{aligned}
$$

the metric is anti-self-dual, with the curvature 2 -forms given by

$$
\begin{aligned}
& \Theta_{01}=-\Theta_{23}=\frac{a}{(r+a)^{3}}\left(-e^{0} \wedge e^{1}+e^{2} \wedge e^{3}\right), \\
& \Theta_{02}=-\Theta_{31}=\frac{a}{(r+a)^{3}}\left(-e^{0} \wedge e^{2}+e^{3} \wedge e^{1}\right), \\
& \Theta_{03}=-\Theta_{12}=\frac{2 a}{(r+a)^{3}}\left(e^{0} \wedge e^{3}-e^{1} \wedge e^{2}\right) .
\end{aligned}
$$

The integrability condition for covariantly-constant spinors therefore implies

$$
\left(\Gamma_{03}-\Gamma_{12}\right) \eta=0
$$

We now make the following Ansatz for a potential $B_{(1)}$ for a harmonic 2-form $G_{(2)}=$ $d B_{(1)}$ :

$$
B_{(1)}=f(d \psi+\cos \theta d \phi),
$$

where $f$ is a function only of $r$. This gives the field strength

$$
G_{(2)}=f^{\prime} d r \wedge(d \psi+\cos \theta d \phi)-f \sin \theta d \theta \wedge d \phi=\frac{f^{\prime}}{2 a} e^{0} \wedge e^{3}-\frac{f}{r^{2}-a^{2}} e^{1} \wedge e^{2} .
$$

Imposing self-duality or anti-self-duality (and thus ensuring that $G_{(2)}$ will be harmonic), we find

$$
f=f_{+} \equiv \frac{r+a}{r-a}, \quad \text { or } \quad f=f_{-} \equiv \frac{r-a}{r+a}
$$

respectively.

The anti-self-dual choice gives a regular 2 -form $G_{(2)}^{-}$, for which

$$
\left(G_{(2)}^{-}\right)^{2}=\frac{4}{(r+a)^{4}}
$$

Clearly $G_{(2)}^{-}$is normalisable, and so in view of the fact that the Taub-NUT manifold is topologically $\mathbb{R}^{4}$, and so trivial, it follows that $G_{(2)}^{-}$must in fact be an exact 2-form. Indeed, we see from (3.29) that if $f=(r-a) /(r+a)$ then $B_{(1)}$ is globally defined, since the coefficient of $(d \psi+\cos \theta d \phi)$ tends appropriately to zero as $r$ approaches $a$. (Note, however, that $B_{(1)}$ itself is not normalisable.) 
Solving for the function $H$ using $L_{(2)}=G_{(2)}^{-}$(3.5) (after first reversing the orientation of the Taub-NUT manifold so that this normalisable harmonic 2-form becomes self-dual and thus satisfies the heterotic equations of motion in the conventions that we are using), we obtain

$$
H=1-\frac{4 a b+m^{2}}{4 a(r-a)}+\frac{m^{2}}{4 a(r+a)} .
$$

If we choose the integration constant $b$ so that $b=-m^{2} /(4 a)$, then the function $H$ becomes non-singular in the entire radial coordinate range, $a \leq r \leq \infty$. The $1 / r$ behaviour of the function $H$ at large $r$ is explained by the fact that the Taub-NUT metric approaches the cylinder $\mathbb{R}^{3} \times S^{1}$. Since $G_{(2)}^{-}$is anti-self-dual, we see that $(3.9)$ is compatible with the condition (3.28) on the covariantly-constant spinors, and so the solution will preserve half the original supersymmetry.

The other possibility is to take $f=f_{+}$in (3.29), in which case we get the self-dual harmonic 2-form

$$
G_{(2)}^{+}=-\frac{1}{(r-a)^{2}}\left(e^{0} \wedge e^{3}+e^{1} \wedge e^{2}\right) .
$$

This has $\left(G_{(2)}^{+}\right)^{2}=4 /(r-a)^{4}$ and so it is clearly non-normalisable. Substituting $L_{(2)}=G_{(2)}^{+}$ into (3.5) we now obtain

$$
H=1-\frac{a m^{2}}{3(r-a)^{3}}-\frac{m^{2}}{2(r-a)^{2}}-\frac{b}{r-a},
$$

and so the solution is clearly singular. Furthermore, since $L_{(2)}$ is now self-dual, it follows that the gaugino criterion (3.9) for supersymmetry is incompatible with (3.28), and so this solution does not preserve any supersymmetry.

\subsection{Heterotic 5-branes on multi Eguchi-Hanson and Taub-NUT instan- tons}

We may also consider the multi Eguchi-Hanson or Taub-NUT metrics [29],

$$
d s_{4}^{2}=V^{-1}\left(d \tau+A_{i} d x_{i}\right)^{2}+V d x_{i} d x_{i},
$$

\footnotetext{
${ }^{4}$ Lest there be confusion about orientation conventions here, we should emphasise again that in order to fit in with the conventions we adopted for the heterotic theory and the Ansatz (3.4) in this paper, which requires that $L_{(2)}$ be self-dual for the solution, we would need to reverse the orientation of the Taub-NUT metric relative to the one given above in which its curvature was anti-self-dual (3.27). This would change the condition 3.28 ) on the covariantly-constant spinor to $\left(\Gamma_{03}+\Gamma_{12}\right) \eta=0$, and the crucial point is that this is compatible with the gaugino condition (3.9) for supersymmetry, $L_{a b} \Gamma^{a b} \epsilon=0$. In order to try to avoid a tedious and repeated re-discussion of this basic issue in later parts of the paper, we shall sometimes tend to speak of using an harmonic form of the "wrong" duality in a solution without labouring the point that one would first need to reverse the orientation of the transverse metric.
} 
where $\partial_{i} \partial_{i} V=0$ and the curvature is self-dual if we take

$$
\partial_{i} V=\epsilon_{i j k} \partial_{j} A_{k}
$$

This solution can describe both $N$ Eguchi-Hanson or $N$ Taub-NUT instantons, where $V$ is chosen as follows:

$$
\begin{aligned}
N \text { Eguchi-Hanson }: & V=\sum_{\alpha=1}^{N+1} \frac{1}{\left|\vec{x}-\vec{x}_{\alpha}\right|}, \\
N \text { Taub-NUT }: & V=1+\sum_{\alpha=1}^{N} \frac{1}{\left|\vec{x}-\vec{x}_{\alpha}\right|} .
\end{aligned}
$$

Let us choose the orthonormal frame $e^{0}=V^{-1 / 2}\left(d \tau+A_{i} d x_{i}\right), e^{i}=V^{1 / 2} d x_{i}$, and make the Ansatz

$$
B_{(1)}=f\left(d \tau+A_{i} d x_{i}\right)
$$

for the potential $B_{(1)}$ for an harmonic 2-form $G_{(2)}=d B_{(1)}$, where $f$ depends only on the three $x_{i}$ coordinates. Then we find

$$
\begin{aligned}
G_{(2)} & =\partial_{i} f d x_{i} \wedge\left(d \tau+A_{i} d x_{i}\right)+\frac{1}{2} f \epsilon_{i j k} \partial_{k} V d x_{i} \wedge d x_{j} \\
& =\partial_{i} f e^{0} \wedge e^{i}+\frac{1}{2} f V^{-1} \partial_{k} V \epsilon_{i j k} e^{i} \wedge e^{j}
\end{aligned}
$$

From this, it follows that $G_{(2)}$ will be self-dual or anti-self-dual (and hence it will be harmonic, since we already know that $\left.d G_{(2)}=0\right)$ if

$$
f=V, \quad \text { or } \quad f=V^{-1}
$$

respectively. Since we have chosen conventions so that the curvature to be self-dual, it follows that the Kähler form will be anti-self-dual. For the two cases, the equation for $H$ in (3.5) becomes

$$
\begin{aligned}
f & =V: & \partial_{i} \partial_{i} H & =-m^{2} V\left(\partial_{i} V\right)^{2} . \\
f & =\frac{1}{V}: & \partial_{i} \partial_{i} H & =-\frac{m^{2}}{V^{3}}\left(\partial_{i} V\right)^{2} .
\end{aligned}
$$

(We refer to footnote 4 for the explanations associated with using the anti-self-dual 2-form.) The solutions are given by

$$
\begin{array}{ll}
f=V: & H=c_{0}+c_{1} V-\frac{1}{6} m^{2} V^{3}, \\
f=\frac{1}{V}: & H=c_{0}+c_{1} V-\frac{m^{2}}{2 V}
\end{array}
$$

where $c_{0}$ and $c_{1}$ are integration constants. 
Of course the harmonic 2-forms that we have constructed here are by no means the only ones that can be found. We can expect that if there are $N+1$ centres in the harmonic function $V$, then there should be in total $N$ independent normalisable (localised) self-dual harmonic 2-forms. (In the context of multi-brane solutions wrapping different supersymmetric two-cycles see [30].) In the case of orbifold construction of K3 manifold, there are sixteen Eguchi-Hanson instantons and hence sixteen localised self-dual harmonic 2-forms. It follows that the most general solution of heterotic 5-branes on K3 can be constructed, in the $T^{4} / Z_{2}$ orbifold limit, as ones located around the sixteen orbifold fixed point with each of the sixteen Cartan 2-form field strengths of Yang-Mills fields equal to the localised self-dual harmonic 2-form of each Eguchi-Hanson instanton, i.e. employing the Ansatz: $F_{(2)}^{i}=m_{i} L_{(2)}^{i}$. Further study of explicit solutions of this type (with both, multi EguchiHanson and Taub-NUT metic) is under way [26].

\section{Dyonic strings on Ricci-flat Kähler 4-manifolds}

The heterotic string admits a compactification to $D=6$ in which the internal fourdimensional manifold is taken to be K3. Various different six-dimensional theories can be obtained, with different Yang-Mills gauge groups, depending upon precisely how the $S U(2)$-valued spin connection of the Ricci-flat Kähler K3 is embedded in the $E_{8} \times E_{8}$ or $S O(32)$ gauge group of the ten-dimensional theory [31]. There will also be quantum corrections to the six-dimensional effective action, whose 1-loop structures can be determined by general arguments based on the necessary anomaly-freedom of the theory. The resulting six-dimensional theories are described by $N=1$ supergravity, coupled to an $N=1$ hypermultiplet and a Yang-Mills multiplet. The bosonic sector comprises the metric $\hat{g}_{\mu \nu}$, a dilaton $\phi$, a 3 -form field strength $F_{(3)}$, and the Yang-Mills fields $G_{(2)}^{a}$. The self-dual part of the 3 -form field belongs to the gravity multiplet, while the anti-self-dual part and the dilaton belong to the hypermultiplet. The field equations [31], including the 1-loop terms, in the language of differential forms that we are using here take the form [32]

$$
\begin{aligned}
\hat{R}_{\mu \nu}= & \frac{1}{2} \partial_{\mu} \phi \partial_{\nu} \phi+\frac{1}{4} e^{-2 \alpha \phi}\left[F_{\mu \nu}^{2}-\frac{1}{6} F_{(3)}^{2} \hat{g}_{\mu \nu}\right] \\
& +\frac{1}{2}\left(v e^{-\alpha \phi}+\tilde{v} e^{\alpha \phi}\right)\left[\left(G^{a}\right)_{\mu \nu}^{2}-\frac{1}{8}\left(G_{(2)}^{a}\right)^{2} \hat{g}_{\mu \nu}\right] \\
d \hat{*} d \phi= & \alpha e^{-2 \alpha \phi} \hat{*} F_{(3)} \wedge F_{(3)}+\frac{1}{2} \alpha\left(v e^{-\alpha \phi}-\tilde{v} e^{\alpha \phi}\right) \hat{*} G_{(2)}^{a} \wedge G_{(2)}^{a}, \\
d\left(e^{-2 \alpha \phi} \hat{*} F_{(3)}\right)= & \frac{1}{2} \tilde{v} G_{(2)}^{a} \wedge G_{(2)}^{a}, \\
D\left[\left(v e^{-\alpha \phi}+\tilde{v} e^{\alpha \phi}\right) \hat{*} G_{(2)}^{a}\right]= & v e^{-2 \alpha \phi} \hat{*} F_{(3)} \wedge G_{(2)}^{a}+\tilde{v} F_{(3)} \wedge G_{(2)}^{a}
\end{aligned}
$$


where $\alpha=1 / \sqrt{2}$, and $D$ denotes the Yang-Mills-covariant exterior derivative. The constants $v$ and $\tilde{v}$ are rational numbers characteristic of the embedding of the $S U(2)$ holonomy group of K3 manifold in the original $E_{8} \times E_{8}$ or $S O(32)$ Yang-Mills gauge group in $D=10$. The terms associated with $\tilde{v}$ come from 1-loop corrections. The field strength $F_{(3)}$ satisfies the Bianchi identity

$$
d F_{(3)}=v G_{(2)}^{a} \wedge G_{(2)}^{a} .
$$

The theory admits a dyonic string solution with the standard type of singular harmonic functions in a flat transverse 4-space. Alternatively, the electric and magnetic string charges can be supplied by a Yang-Mills instanton living in the transverse 4-space [33], thus giving a non-singular solution. Here, we shall show that there is another way to obtain a nonsingular dyonic string solution, by instead considering a 4-space with a non-trivial self-dual 2-form, and with the charges now supplied by a $U(1)$ Abelian gauge field contained within the Yang-Mills fields. We consider the following Ansatz for the dyonic string

$$
\begin{aligned}
d \hat{s}_{6}^{2} & =\left(H_{1} H_{2}\right)^{-1 / 2}\left(-d t^{2}+d x^{2}\right)+\left(H_{1} H_{2}\right)^{1 / 2} d s_{4}^{2}, \\
F_{(3)} & =d t \wedge d x \wedge d H_{1}^{-1}+* d H_{2}, \\
\phi & =\alpha \log \left(H_{2} / H_{1}\right), \quad G_{(2)}=m L_{(2)},
\end{aligned}
$$

Note that here $G_{(2)}$ is an Abelian gauge field taken from the original Yang-Mills fields $G_{(2)}^{\alpha}$. It is straightforward to verify that all the equations of motion are satisfied provided that $L_{(2)}$ is an harmonic self-dual 2 -form in the transverse 4-metric $d s_{4}^{2}$, and that $H_{1}$ and $H_{2}$ satisfy

$$
\square H_{1}=-\frac{1}{4} \tilde{v} m^{2} L_{(2)}^{2}, \quad \square H_{2}=-\frac{1}{4} v m^{2} L_{(2)}^{2} .
$$

The solutions for $H_{1}$ and $H_{2}$ are the same form as the ones we found for the function $H$ for the heterotic 5-brane. Here, we shall consider the dyonic string on the Eguchi-Hanson metric (3.10). The non-singular solutions for $H_{1}$ and $H_{2}$ are then given by

$$
H_{1}=1+\frac{\tilde{v} m^{2}}{2 a^{2} r^{2}}, \quad H_{2}=1+\frac{v m^{2}}{2 a^{2} r^{2}}
$$

In the case of the heterotic string compactified on K3 manifold, the value of $v$ or $\tilde{v}$ can be negative such that the dyonic string becomes massless [33], which indicates a phase transition [34, 33]. Such a solution is usually associated with a naked singularity in the region where $H_{1}$ or $H_{2}$ vanishes. Singularities of this type are of the repulson type [35], and their resolution via an "enhançon" mechanism [36] was proposed. Interestingly, our new resolved dyonic string solution on the Eguchi-Hanson metric avoids the repulson singularity if $a$ is taken to be sufficiently large, thus providing an alternative to the resolution via the 
enhançonmechanism. This way of avoiding the repulson singularity is somewhat similar to the way in which it can be avoided for the dyonic string supported by a Yang-Mills instanton of sufficiently large scale size [33, 32].

In an appropriate decoupling limit, the constant 1 in the functions $H_{1}$ and $H_{2}$ can be dropped, and the resulting dyonic string can be dimensionally reduced to give a $D=3$ domain-wall solution. For simplicity, let us consider the case where $H_{1}=H_{2} \longrightarrow R^{2} / r^{2}$. It follows that the $D=3$ domain wall is given by

$$
d s_{3}^{2}=\frac{r^{2}}{R^{2}} W\left(-d t^{2}+d x^{2}\right)+\frac{R^{2} d r^{2}}{r^{2}} .
$$

Note that the metric is asymptotically $\mathrm{AdS}_{3}$ at $r \rightarrow \infty$, implying that the solution is supported by a non-trivial scalar potential in $D=3$ with a fixed point. The metric can be transformed to the conformally-flat frame $d s_{3}^{2}=e^{2 A(z)}\left(-d t^{2}+d x^{2}+d z^{2}\right)$, by means of the coordinate transformation $z=\left(R^{2} / r\right){ }_{2} F_{1}\left[1 / 4,1 / 2 ; 5 / 4 ; a^{4} / r^{4}\right]$, and hence $z$ runs from some negative value $z^{*}$ to $z=0$. The Schrödinger potential for the minimally-coupled scalar has the following behaviour

$$
\begin{aligned}
z \rightarrow z^{*}: & V=-\frac{3}{16\left(z-z^{*}\right)^{2}}, \\
z \rightarrow 0: & V=\frac{3}{4 z^{2}}
\end{aligned}
$$

and so the spectrum (describing the dual three-dimensional field theory in the infra-red) is discrete, with positive-definite energy. Note that the energy level separation is governed by the the ratio $m^{2} / a^{2}$.

\section{M2-branes on 8-manifolds}

\subsection{General discussion}

The bosonic section of eleven-dimensional supergravity comprises the metric and a 3 -form potential, with the Lagrangian given by

$$
\mathcal{L}_{\mathrm{M}}=\hat{R} \hat{*} \mathbb{1}-\frac{1}{2} \hat{*} F_{(4)} \wedge F_{(4)}+\frac{1}{6} F_{(4)} \wedge F_{(4)} \wedge A_{(3)},
$$

where $F_{(4)}=d A_{(3)}$. The equation of motion for the $A_{(3)}$ is given by

$$
d \hat{*} F_{(4)}=\frac{1}{2} F_{(4)} \wedge F_{(4)}
$$

The theory admits an M2-brane solution, which has an 8-dimensional transverse space. The equation of motion (5.2) suggests that the M2-brane charge can be supported by a 
non-trivial harmonic 4-form in the 8-dimensional transverse space. This motivates us to make the following generalisation of the usual M2-brane Ansatz:

$$
\begin{aligned}
d \hat{s}_{11}^{2} & =H^{-2 / 3} d x^{\mu} d x^{\nu} \eta_{\mu \nu}+H^{1 / 3} d s_{8}^{2} \\
F_{(4)} & =d^{3} x \wedge d H^{-1}+m L_{(4)}
\end{aligned}
$$

where $L_{(4)}$ is an harmonic 4-form in the transverse 8-manifold $M_{8}$, which has a Ricci-flat metric $d s_{8}^{2}$. Clearly the Bianchi identity $d F_{(4)}$ is trivially satisfied, since we have $d L_{(4)}=0$. The equation of motion (5.2) implies

$$
\square H=-\frac{1}{48} m^{2} L_{(4)}^{2}, \quad L_{4}=* L_{(4)} .
$$

where $*$ is the Hodge dual with respect to $d s_{8}^{2}$, and one can then easily verify that the Einstein equation is also satisfied, provided that (5.4) is satisfied.f Next, we shall consider an M2-brane on an explicit example of a complete non-compact 8-manifold of Spin(7) holonomy that has a non-trivial normalisable self-dual harmonic 4-form. We shall see that we can obtain a non-singular M2-brane solution.

\subsection{M2-brane on 8-manifold of $\operatorname{Spin}(7)$ holonomy}

There are many examples of Ricci-flat 8-manifolds, including those with hyper-Kähler and Kähler metrics. Another possibility is to consider 8-manifolds with $\operatorname{Spin}(7)$ holonomy; this is one of the exceptional cases included in Berger's classification [14]. There is a simple construction for one such example of a complete Ricci-flat 8-manifold with Spin(7) holonomy [15, 16]. The metric has cohomogeneity one, with the level surfaces being 7 -spheres described as a principal $S U(2)$ bundle over $S^{4}$ :

$$
d s_{8}^{2}=\alpha^{2} d r^{2}+\beta^{2}\left(\sigma_{i}-A^{i}\right)^{2}+\gamma^{2} d \Omega_{4}^{2}
$$

where $\alpha, \beta$ and $\gamma$ are functions of $r$, and as usual $\sigma_{i}$ are left-invariant 1-forms of $S U(2)$. The $S U(2)$ Yang-Mills potentials $A^{i}$ describe the BPST instanton on the unit 4-sphere whose metric is $d \Omega_{4}^{2}$. One finds that the metric is Ricci flat if [15, 16]

$$
\alpha^{2}=\left(1-\frac{a^{10 / 3}}{r^{10 / 3}}\right)^{-1}, \quad \beta^{2}=\frac{9}{100} r^{2}\left(1-\frac{a^{10 / 3}}{r^{10 / 3}}\right), \quad \gamma^{2}=\frac{9}{20} r^{2} .
$$

The radial coordinate runs from $r=a$ to $r=\infty$. At $r=a$, the metric smoothly approaches $R^{4} \times S^{4}$, whilst at large $r$ the level surfaces tend to the homogeneous squashed Einstein metric on $S^{7}$. Topologically, the manifold is an $R^{4}$ bundle over $S^{4}$.

\footnotetext{
${ }^{5} \mathrm{M} 2$-brane solutions with a non-normalisable self-dual 4-form in the usual flat transverse 8-space were constructed in [37]; these were not supersymmetric, and they had naked singularities.
} 
In order to look for a self-dual harmonic 4-form, it is useful to introduce the quantities

$$
\epsilon_{(3)} \equiv \nu_{1} \wedge \nu_{2} \wedge \nu_{3}, \quad X_{(3)} \equiv \nu_{i} \wedge F^{i}, \quad Y_{(4)} \equiv \frac{1}{2} \epsilon_{i j k} \nu_{i} \wedge \nu_{j} \wedge F^{k}
$$

where $\nu_{i} \equiv \sigma_{i}-A^{i}$, and $F^{i} \equiv d A^{i}+\frac{1}{2} \epsilon_{i j k} A^{j} \wedge A^{k}$ is the Yang-Mills field strength for the BPST instanton. We have that $F^{i}$ is self-dual in the unit 4-sphere metric $d \Omega_{4}^{2}$, and $F^{i} \wedge F^{i}=6 \Omega_{(4)}$, where $\Omega_{(4)}$ is the volume form of the unit 4-sphere. In terms of these, we make the following Ansatz for a 3 -form potential $B_{(3)}$ from which we shall seek to construct a self-dual or anti-self-dual harmonic 4 -form $G_{(4)}=d B_{(3)}$ :

$$
B_{(3)}=f \epsilon_{(3)}+g X_{(3)}
$$

where $f$ and $g$ are functions only of $r$. Thus we find

$$
G_{(4)}=f^{\prime} d r \wedge \epsilon_{(3)}-(f+g) Y_{(4)}+g^{\prime} d r \wedge X_{(3)}-6 g \Omega_{(4)} .
$$

Hodge dualisation in the metric (5.5) gives

$$
*\left(d r \wedge \epsilon_{(3)}\right)=\frac{\gamma^{4}}{\alpha \beta^{3}} \Omega_{(4)}, \quad \tilde{Y}_{(4)}=\frac{\alpha}{\beta} d r \wedge X_{(3)} .
$$

From this, it follows that imposing the duality condition $\tilde{G}_{(4)}=\eta G_{(4)}$, where $\eta= \pm 1$, gives the equations

$$
f^{\prime}=-6 \eta g \alpha \beta^{3} \gamma^{-4}, \quad g^{\prime}=-\eta \alpha \beta^{-1}(f+g),
$$

where $\eta= \pm 1$ corresponds respectively to self-duality and anti-self-duality. Of course since $G_{(4)}$ by construction is closed, it follows that after imposing (anti) self duality, it will be harmonic.

Defining $z=(a / r)^{10 / 3}$, these equations can be solved to give

$$
\begin{aligned}
\text { self-dual : } & g=\frac{c_{1} z^{-1 / 5}}{1-z}+\frac{c_{2} z^{6 / 5}}{1-z}, \quad f=-\frac{6}{5} c_{1} z^{-1 / 5}+\frac{1}{5} c_{2} z^{6 / 5}, \\
\text { anti-self-dual : } & g=c_{1} z^{-6 / 5}+c_{2} z^{1 / 5}, \quad f=\frac{1}{5} c_{1}(1-6 z) z^{-6 / 5}+\frac{1}{5} c_{2}(z-6) z^{1 / 5} .
\end{aligned}
$$

For suitable choices of the constants, it can be arranged that the self-dual harmonic 4form $G_{(4)}$ has a non-diverging magnitude at $r=a$, but it then does not fall off fast enough at $r=\infty$ to be square integrable.

On the other hand, the anti-self-dual harmonic 4-form $G_{(4)}^{-}$has magnitude given by the simple expression

$$
\left(G_{(4)}^{-}\right)^{2}=\frac{71680000 c_{1}^{2}}{243 a^{8}}+\frac{35840000 a^{4 / 3} c_{2}^{2}}{729 r^{28 / 3}},
$$

which is non-diverging at $r=a$ for all choices of its $c_{1}$ and $c_{2}$ integration constants. In fact the case $c_{2}=0$ just corresponds to the covariantly-constant harmonic 4-form that 
characterises a manifold of $\operatorname{Spin}(7)$ holonomy; it can be expressed as $G_{a b c d}=\bar{\eta} \Gamma_{a b c d} \eta$ where $\eta$ is the covariantly-constant spinor (see [16] for further details). Accordingly, the case of greater interest to us is when $c_{1}=0$ and $c_{2}=1$, so that we get a normalisable harmonic 4-form. Expressed back in terms of $r$, this solution is given by

$$
f=\frac{1}{5}\left(\frac{a}{r}\right)^{2 / 3}\left(\frac{a^{10 / 3}}{r^{10 / 3}}-6\right), \quad g=\left(\frac{a}{r}\right)^{2 / 3}
$$

From this, it follows that

$$
\left(G_{(4)}^{-}\right)^{2}=\frac{35840000 a^{4 / 3}}{729 r^{28 / 3}} .
$$

It is easily seen that this is square-integrable. It is topologically non-trivial, since the expression for $B_{(3)}$ becomes singular at $r=a$. (This can be seen from the fact that the coefficients of $\epsilon_{(3)}$ and $X_{(3)}$ fail to vanish at $r=a$.) In other words, it cannot be written globally as the exterior derivative of a 3 -form, and so it is closed but not exact.

If we take $L_{4}=G_{(4)}^{-}$in (5.4), we get the first integral

$$
\frac{\beta^{3} \gamma^{4}}{\alpha} H^{\prime}=b+\frac{3 a^{4 / 3} m^{2}\left(7 r^{10 / 3}-2 a^{10 / 3}\right)}{5 r^{14 / 3}}
$$

where $b$ is a constant. (Again we refer to footnote 4 for a discussion of the necessary orientation reversal.) The remaining integration can be performed explicitly, giving an expression for $H$ in terms of elementary functions. For generic values of the constant $b$, the function $H$ diverges like $1 /(r-a)$ as $r$ approaches $a$, but this can be eliminated by choosing $b=-3 m^{2}$. After doing this, we find that $H$ is regular everywhere in the interval $a \leq r \leq \infty$, and it is given by

$$
\begin{array}{r}
H=c-\frac{40000 m^{2}}{729 a^{16 / 3} r^{2 / 3}}\left[9-\left(\frac{a}{r}\right)^{10 / 3}+\frac{3\left(1-\frac{a^{2}}{r^{2}}\right)}{1-\left(\frac{a}{r}\right)^{10 / 3}}\right] \\
+\frac{32000 \sqrt{2 \sqrt{5}} m^{2}}{243 a^{6}}\left[(\sqrt{5}-1) \arctan \left(\frac{\sqrt{5}+1+4\left(\frac{a}{r}\right)^{10 / 3}}{\sqrt{2 \sqrt{5}(\sqrt{5}-1)}}\right)\right. \\
\left.+(\sqrt{5}+1) \arctan \left(\frac{\sqrt{5}-1+4\left(\frac{a}{r}\right)^{10 / 3}}{\sqrt{2 \sqrt{5}(\sqrt{5}+1)}}\right)\right] .
\end{array}
$$

At large $r, H$ has the following behaviour,

$$
H=c+\frac{210^{5} m^{2}}{3^{7} r^{6}}-\frac{2810^{4} a^{4 / 3} m^{2}}{2673 r^{22 / 3}}+\cdots
$$

It should be emphasised that the Chern-Simons flux term plays a crucial rule for obtaining this regular M2-brane solution; the singularity would become unavoidable if we were to set $m=0$ in (5.16). 
We may also consider the solution for an M2-brane supported by the self-dual solution for $G_{(4)}$, given by (5.12). If we choose the constant $c_{1}=0$, then we have the following

$$
r \rightarrow a: \quad\left(G_{(4)}^{+}\right)^{2} \sim \frac{\text { const }}{(\mathrm{r}-\mathrm{a})^{4}}, \quad r \rightarrow \infty: \quad\left(G_{(4)}^{+}\right)^{2} \sim \frac{\text { const }}{\mathrm{r}^{16}}
$$

and so it is normalisable at $r \rightarrow \infty$, but the integral $\int \sqrt{g} d^{8} y\left(G_{(4)}^{+}\right)^{2}$ diverges at $r \rightarrow a$. The solution for the function $H$ has the following asymptotic behaviour:

$$
\begin{aligned}
r \rightarrow a: & H \sim-\frac{\text { const }}{(\mathrm{r}-\mathrm{a})^{3}} \rightarrow-\infty, \\
r \rightarrow \infty: & H \sim 1+\frac{Q}{r^{6}}+\frac{\text { const }}{\mathrm{r}^{14}} .
\end{aligned}
$$

The solution is well-behaved for $r \rightarrow \infty$, with a well-defined ADM mass, but it has a naked singularity when $r$ approaches $a$. In order to avoid such a naked singularity, we can instead choose the constants so that $c_{1}=-c_{2}$, in which case the harmonic 4-form has the following asymptotic behaviour:

$$
r \rightarrow a: \quad\left(G_{(4)}^{+}\right)^{2} \sim \text { const. }, \quad r \rightarrow \infty: \quad\left(G_{(4)}^{+}\right)^{2} \sim \frac{\text { const }}{\mathrm{r}^{20 / 3}} .
$$

This is normalisable in the region $r \rightarrow a$, but non-normalisable for $r \rightarrow \infty$. The function $H$ is given by

$$
\begin{aligned}
H= & c+\frac{1600 m^{2} c_{2}^{2} y^{2}}{729 a^{6}\left(1+y+y^{2} y^{3}+y^{4}\right)^{3}}\left(42+126 y+231 y^{2}+357 y^{3}+504 y^{4}+633 y^{5}\right. \\
& +744 y^{6}+809 y^{7}+828 y^{8}+801 y^{9}+700 y^{10}+525 y^{11}+375 y^{12}+250 y^{13}+150 y^{14} \\
& \left.+75 y^{15}+25 y^{16}\right)-\frac{44800 m^{2} c_{2}^{2}}{243 a^{6}} \sum_{i=i}^{4} \frac{y_{i} \log \left(y-y_{i}\right)+y_{i}^{2} \log \left(y-y_{i}\right)}{1+2 y_{i}+3 y_{i}^{2}+4 y_{i}^{3}}
\end{aligned}
$$

where $y=(a / r)^{2 / 3}$ and $y_{i}$ are the four roots of the polynomial $1+y+y^{2}+y^{3}+y^{4}=0$. It is easy to verify then that $H$ becomes a constant at $r=a$, and behaves like $H \sim$ $c+$ const $/ r^{14 / 3}+Q / r^{6}$ at large $r$. Thus we see that $H$ does not fall off fast enough to give a well-defined ADM mass in this case, which is a consequence of the fact that $G_{(4)}$ is not normalisable asymptotically. However, the solution is regular everywhere.

\section{D2-branes on 7-manifolds}

\subsection{General discussion}

The D2-brane is supported by the 3-form potential in type IIA theory. It has a 7-dimensional transverse space. At first sight, one might think that the D2-brane is nothing but the vertical 
dimensional reduction of the M2-brane we discussed above. However, we can have a nontrivial 7-dimensional space that is not merely the $S^{1}$ reduction of one of the 8-dimensional spaces of the kind discussed in the previous section, and so new kinds of deformed solution are possible here. The bosonic Lagrangian for type IIA supergravity is given by

$$
\begin{aligned}
\mathcal{L}_{\mathrm{IIA}}= & \hat{R} \hat{*} \mathbb{1}-\frac{1}{2} \hat{*} d \phi \wedge d \phi-\frac{1}{2} e^{-\phi} \hat{*} F_{(3)} \wedge F_{(3)}-\frac{1}{2} e^{\frac{1}{2} \phi} \hat{*} F_{(4)} \wedge F_{(4)}-\frac{1}{2} e^{\frac{3}{2} \phi} \hat{*} F_{(2)} \wedge F_{2} \\
& +\frac{1}{2} d A_{(3)} \wedge d A_{(3)} \wedge A_{(2)},
\end{aligned}
$$

where

$$
F_{4}=d A_{3}+A_{(2)} \wedge d A_{(1)}, \quad F_{(3)}=d A_{(2)}, \quad F_{(2)}=d A_{(1)} .
$$

We shall look for a D2-brane solution for which $A_{(1)}$ vanishes, and hence we shall have $d F_{(4)}=0$. The equation of motion for $A_{(3)}$ is given by

$$
d\left(e^{\frac{1}{2} \phi} \hat{*} F_{(4)}\right)=F_{(4)} \wedge F_{(3)}
$$

Thus if we can have a non-trivial harmonic 3-form in the 7-dimensional transverse space, we can arrange to have a non-vanishing Chern-Simons term, supporting the D2-brane.

Let us consider the following D2-brane Ansatz

$$
\begin{aligned}
d \hat{s}_{10}^{2} & =H^{-5 / 8} d x^{\mu} d x^{\nu} \eta_{\mu \nu}+H^{3 / 8} d s_{7}^{2}, \\
F_{4} & =d^{3} x \wedge d H^{-1}+m_{1} * L_{(3)}, \quad F_{3}=m_{2} L_{(3)}, \quad \phi=\frac{1}{4} \log H,
\end{aligned}
$$

where $L_{(3)}$ is an harmonic 3-form in the Ricci-flat 7-metric $d s_{7}^{2}$. It is straightforward to verify that all the type IIA equations of motion are then satisfied provided that $m_{1}=-m_{2} \equiv m$, and that

$$
\square H=-\frac{1}{6} m^{2} L_{(3)}^{2}
$$

\subsection{D2-brane on 7-manifold with $G_{2}$ holonomy}

We may consider a simple example of a complete non-compact 7-manifold with a Ricci-flat metric of $G_{2}$ holonomy. It is given by [15, 16]

$$
d s_{7}^{2}=\alpha^{2} d r^{2}+\beta^{2}\left(\sigma_{i}-\frac{1}{2} \Sigma_{i}\right)^{2}+\gamma^{2} \Sigma_{i}^{2}
$$

where the functions $\alpha, \beta$ and $\gamma$ are given by

$$
\alpha^{2}=\left(1-\frac{a^{3}}{r^{3}}\right)^{-1}, \quad \beta^{2}=\frac{1}{9} r^{2}\left(1-\frac{a^{3}}{r^{3}}\right), \quad \gamma^{2}=\frac{1}{12} r^{2} .
$$

Here $\Sigma_{i}$ and $\sigma_{i}$ are two sets of left-invariant 1-forms on two independent $S U(2)$ group manifolds. The level surfaces $r=$ constant are therefore $S^{3}$ bundles over $S^{3}$. This bundle 
is trivial, and so in fact the level surfaces are topologically $S^{3} \times S^{3}$. The radial coordinate runs from $r=a$ to $r=\infty$.

If we define an orthonormal frame by

$$
e^{0}=\alpha d r, \quad e^{i}=\gamma \Sigma_{i}, \quad e^{i+3}=\beta \nu_{i}
$$

where $\nu_{i} \equiv \sigma_{i}-\frac{1}{2} \Sigma_{i}$, then one can read off from results in 16 that there is a single covariantly-constant spinor, which satisfies the projection conditions

$$
\left(\Gamma_{04}-\Gamma_{23}\right) \eta=\left(\Gamma_{05}-\Gamma_{31}\right) \eta=\left(\Gamma_{06}-\Gamma_{12}\right) \eta=0
$$

As discussed in [16], one can then construct a covariantly-constant 3-form $Q_{(3)}$, defined by $Q_{a b c}=\bar{\eta} \Gamma_{a b c} \eta$. This turns out to be

$$
Q_{(3)}=e^{0} \wedge e^{i} \wedge e^{\tilde{i}}+\frac{1}{2} \epsilon_{i j k} e^{i} \wedge e^{\tilde{j}} \wedge e^{\tilde{k}}-e^{1} \wedge e^{2} \wedge e^{3}
$$

where we have defined $e^{\tilde{i}} \equiv e^{i+3}=\beta \nu_{i}$ for $i=1,2,3$.

The form of $Q_{(3)}$ suggests a natural Ansatz for trying to find further harmonic 3-forms. Thus we let

$$
G_{(3)}=f d r \wedge \nu_{i} \wedge \Sigma_{i}+\frac{1}{2} g \epsilon_{i j k} \nu_{i} \wedge \nu_{j} \wedge \Sigma_{k}+h \Sigma_{1} \wedge \Sigma_{2} \wedge \Sigma_{3}
$$

where $f, g$ and $h$ are functions only of $r$. The condition $d G_{(3)}=0$ implies

$$
4 h^{\prime}-3 f=0, \quad g^{\prime}+f=0,
$$

giving a first integral

$$
h=3 b-\frac{3}{4} g,
$$

where $b$ is an arbitrary constant. The condition $d * G_{(3)}=0$ gives the equation

$$
\left(\frac{f \beta \gamma}{\alpha}\right)^{\prime}+\frac{g \alpha \gamma}{\beta}-\frac{h \alpha \beta^{3}}{4 \gamma^{3}}=0
$$

We find that the general solution is

$$
g=\frac{b\left(r^{3}-4 a^{3}\right)+c_{1} / r+c_{2} r^{3}\left(4 r^{3}-7 a^{3}\right)}{r^{3}-a^{3}},
$$

together with

$$
f=-g^{\prime}, \quad h=3 b-\frac{3}{4} g .
$$

The magnitude of $G_{(3)}$ is given by

$$
G_{(3)}^{2}=6\left(\frac{3 f^{2}}{\alpha^{2} \beta^{2} \gamma^{2}}+\frac{3 g^{2}}{\beta^{4} \gamma^{2}}+\frac{h^{2}}{\gamma^{6}}\right) .
$$


It follows that $G_{(2)}^{2}$ will diverge at $r=a$ in general, but it will be non-singular if the constants are chosen so that

$$
c_{1}=3 a^{4}\left(b+a^{3} c_{2}\right)
$$

The original covariantly-constant 3-form $Q_{(3)}$ is obtained if one additionally sets $b=-a^{3} c_{2}$. In this case we would find that $G_{(3)}^{2}$ was simply a constant. Instead, we can get a harmonic 3 -form that falls off at large $r$ if we still impose (6.18), but now additionally choose $c_{2}=0$. This gives a harmonic 3-form with the following asymptotic behaviours:

$$
G_{(3)}^{2} \sim \text { const }+(\text { const })(r-a)+\cdots
$$

as $r$ approaches $a$, and

$$
G_{(3)}^{2} \sim \frac{\text { const }}{r^{6}}+O\left(1 / r^{9}\right)
$$

as $r$ approaches infinity. This is almost, but not quite, normalisable, for $r \rightarrow \infty$. The function $H$ can be solved explicitly, given by

$$
\begin{aligned}
H= & c+\frac{108 m^{2} b^{2}(a+r)}{a^{3} r^{3}\left(r^{2}+a r+a^{2}\right)^{3}}\left(16 r^{7}+24 a r^{6}+48 a^{2} r^{5}+47 a^{3} r^{4}+54 a^{4} r^{3}\right. \\
& \left.+36 a^{5} r^{2}+18 a^{6} r+9 a^{7}\right)+1152 \sqrt{3} m^{2} b^{2} a^{-4} \arctan \frac{2 r+a}{\sqrt{3} a} .
\end{aligned}
$$

Thus the function $H$ is perfectly non-singular for $r$ running from $a$ to infinity. It approaches a positive constant for $r \rightarrow a$, with the asymptotic behaviour

$$
H \sim \text { const }+\frac{\text { const }}{\mathrm{r}^{4}}+\frac{\text { const }}{\mathrm{r}^{5}},
$$

for $r \rightarrow \infty$. Thus the function $H$ does not fall off fast enough to have a well-defined $\mathrm{ADM}$ mass, which is a consequence of the fact that $G_{(3)}^{2}$ is linearly non-normalisable in the asymptotic region.

\section{Further examples}

\subsection{Type IIA and type IIB strings on 8-manifolds}

There are two different possibilities for obtaining deformed string solutions in ten dimensions, depending upon whether we consider type IIA strings or type IIB strings. In each case, the 8-dimensional transverse space will first be replaced by a Ricci-flat manifold $M_{8}$.

In type IIA, the string can be obtained as a diagonal dimensional reduction of the eleven-dimensional M2-brane. Since this leaves the transverse space intact, the deformed 
solution follows directly from our results for the deformed M2-brane in section 5. Thus the solution is given by

$$
\begin{aligned}
d \hat{s}_{10}^{2} & =H^{-3 / 4}\left(-d t^{2}+d x^{2}\right)+H^{1 / 4} d s_{8}^{2}, \\
F_{(3)} & =d t \wedge d x \wedge d H^{-1}, \quad \phi=-\frac{1}{2} \log H, \\
F_{(4)} & =m L_{(4)}, \quad F_{(2)}=0 .
\end{aligned}
$$

This satisfies the equations of motion of type IIA supergravity provided that $d s_{8}^{2}$ is a Ricciflat on the transverse space $M_{8}, L_{(4)}$ is a self-dual harmonic 4-form on $M_{8}$, and $H$ satisfies

$$
\square H=-\frac{1}{48} m^{2} L_{(4)}^{2} .
$$

(See section 6 for the convention and the Lagrangian of type IIA supergravity.) All the features of the deformed M2-brane solutions will carry over directly to these deformed type IIA string solutions. The resulting type IIA string is then completely regular, as in the case of M2-brane.

The situation is quite different if we consider strings in the type IIB theory instead. Now, for an NS-NS string, the Ansatz will be

$$
\begin{aligned}
& d \hat{s}_{10}^{2}=H^{-3 / 4}\left(-d t^{2}+d x^{2}\right)+H^{1 / 4} d s_{8}^{2}, \\
& F_{(3)}^{\mathrm{NS}}=d t \wedge d x \wedge d H^{-1}, \quad \phi=-\frac{1}{2} \log H, \\
& F_{(3)}^{\mathrm{RR}}=m L_{(3)}, \quad F_{(5)}=m * L_{(3)}+m H^{-1} d t \wedge d x \wedge L_{(3)},
\end{aligned}
$$

where $d s_{8}^{2}$ is a Ricci-flat 8-metric on a manifold $M_{8}$ and $L_{(3)}$ is an harmonic 3-form on $M_{8}$.

The notation for the type IIB fields is the same as in section 2 , with $F_{(3)}^{\mathrm{RR}}$ denoting the R-R 3-form, and $F_{(3)}^{\mathrm{NS}}$ denoting the NS-NS 3-form.

Substituting into the type IIB equations of motion, (given in section 2,) we find that the above Ansatz for a deformed NS-NS string solve the type IIB equations of motion, provided that $L_{(3)}$ is harmonic and that $H$ satisfies

$$
\square H=-\frac{1}{6} m^{2} L_{(3)}^{3} .
$$

If there is a normalisable 3 -form in $M_{8}$ then it will be possible to construct a string solution with no singularities. As usual, if the manifold $M_{8}$ has a special holonomy, so that it admits covariantly-constant spinors, then the string solution can still preserve some supersymmetry.

\subsection{D4-branes on 5-manifolds}

Another example that can be constructed is a deformed D4-brane solution in the type IIA theory. In this case the transverse space is five-dimensional. There are no irreducible 
manifolds $M_{5}$ of special holonomy, but for completeness we may consider this example too. Thus we make the Ansatz

$$
\begin{aligned}
d \hat{s}_{10}^{2} & =H^{-3 / 8} d x^{\mu} d x^{\nu} \eta_{\mu \nu}+H^{5 / 8} d s_{5}^{2}, \\
F_{(4)} & =* d H, \quad \phi=-\frac{1}{4} \log H \\
F_{(2)} & =m L_{(2)}, \quad F_{(3)}=m * L_{(2)} .
\end{aligned}
$$

Substituting into the equations of motion of type IIA supergravity, (given in section 6,) we find that this gives a deformed D4-brane solution provided that $L_{(2)}$ is an harmonic 2-form in the manifold $M_{5}$ with Ricci-flat metric $d s_{5}^{2}$, and that $H$ satisfies

$$
\square H=-\frac{1}{2} m^{2} L_{(2)}^{2}
$$

One possible choice for $M_{5}$ is to take the product $M_{5}=M_{4} \times \mathbb{R}$, where $M_{4}$ is any Ricciflat 4-manifold. For example, we can take $M_{(4)}$ to be Eguchi-Hanson (Taub-NUT) metric, in which case the self-dual (anti-self-dual) harmonic 2-form given in (3.15) will also be harmonic in $M_{5}$. It will no longer be normalisable, owing to the non-compact 5 'th direction upon which it does not depend. One could take the $5^{\prime}$ th direction to be $S^{1}$ instead, in which case it would still be normalisable in $M_{5}=M_{4} \times S^{1}$.

\section{Conclusions}

In this paper we have introduced a general procedure for obtaining families of deformations of certain of the standard $p$-brane solutions in supergravity. The method is applicable to cases where the field strength that supports the standard brane solution has ChernSimons type terms (or transgressions) in its Bianchi identity or equation of motion. The deformed solution is obtained by first replacing the flat transverse space by a manifold $M_{n}$ with a Ricci-flat metric, and then using a harmonic form in $M_{n}$ to give a non-vanishing flux, which introduces fractional branes, for the fields appearing bilinearly in the ChernSimons terms. The cases of principle interest are where $M_{n}$ admits covariantly-constant spinors, since means that the deformed solution may still preserve some supersymmetry, thus providing gravity solutions that are dual to super-Yang-Mills theories, possibly with less than maximal supersymmetry. Usually, one would also want to take $M_{n}$ to be a complete non-compact manifold. If the relevant harmonic form is normalisable (square integrable), then the deformed brane solution can become completely free of singularities. These examples are of special interest since now the supergravity description is valid in the whole domain of space-time and thus can provide important information about the 
properties of the dual field theory (in the infra-red regime), such as confinement and chiral symmetry breaking.

The method that we have developed here for obtaining the deformed brane solutions is a generalisation of a procedure that has been much discussed recently in the context of D3branes, in which non-zero flux for the R-R and NS-NS 3-forms (fractional branes) is turned on [1, 2, 3, 5]. These examples are of special interest, since they provide information on strongly coupled four-dimensional $N=1$ super-Yang-Mills theory. One of the resolutions of the D3-brane is discussed in [5]. In this particular case the harmonic 3-form used in the deformed solution is not normalisable, and so the naked singularity, of the repulson-type 35, 36, in the D3-brane solution is not eliminated.

In our more general discussion for a variety of other dimensions, we found several explicit examples where brane singularities could be completely resolved, including the heterotic 5brane, the dyonic string, the M2-brane, the type IIA string and the D2-brane. In general, such a complete resolution can be achieved whenever one has a normalisable harmonic form to supply the required flux.

A particularly interesting example is that of heterotic 5-brane, where the resolution is achieved by taking an Abelian $U(1)$ gauge field to have a flux proportional to the normalisable self-dual (or anti-self-dual) harmonic 2-form of the Eguchi-Hanson (or Taub-NUT) metric. Since the Eguchi-Hanson metrics can provide local resolutions of the $T^{4} / Z_{2}$ orbifold singularities, these examples provide a completely non-singular solution of the 5-brane on the K3 manifold, with each of the sixteen 2-form field strengths in the Cartan subalgebra of the heterotic string equal to the localised self-dual harmonic 2-form of each Eguchi-Hanson instanton. The non-singular gravity solution may provide a viable candidate for studying the dual six-dimensional field theory with $N=2$ supersymmetry. A preliminary analysis indicates that the spectrum may not have a bound state, and that it is continuous with a mass gap.

The dyonic string is another example of a completely regular deformed solution, whose three-dimensional field-theory dual exhibits a bound-state spectrum and thus a confinement. Interestingly, the example of the tensionless string, which exhibits a repulson-type singularity [35], can now be completely resolved, yielding a non-singular solution in the whole region, thus providing an alternative to Yang-Mills instanton resolution[33] and the enhançon resolution [36.

There are a number of possible generalisations [26] of the brane-resolution mechanism constructed in this paper, which may involve more then one harmonic (normalisable) form 
as well as possible intersecting $p$-brane configurations. These would provide novel regular supergravities as candidate duals for field theories with less supersymmetry, and in particular, the $N=1$ supersymmetric four-dimensional examples.

\section{Acknowledgement}

We are very grateful to Gary Gibbons for extensive discussions about non-compact Ricciflat metrics, and to Leopoldo Pando-Zayas for useful discussion on D3-branes on conifolds. We also benefited from discussions with Christian Römelsberger and Barthomeu Fiol. We thank Rutgers High Energy Theory Group (M.C.) and the organisers of the "Semestres Cordes" at the Centre Emile Borel of the Institut Henri Poincaré (C.N.P.) for support and hospitality during the course of this work.

\section{References}

[1] I.R. Klebanov and A.A. Tseytlin, Gravity duals of supersymmetric $S U(N) \times S U(N+m)$ gauge theories, Nucl. Phys. B578 (2000) 123, hep-th/0002159.

[2] I.R. Klebanov and M.J. Strassler, Supergravity and a confining gauge theory: duality cascades and $\chi S B$-resolution of naked singularities, JHEP 0008:052,2000, hepth/0007191.

[3] M. Graña and J. Polchinski, Supersymmetric three-form flux perturbations on $A d S_{5}$, hep-th/0009211.

[4] S. Gubser, Supersymmetry and F-theory realization of the deformed conifold with threeform flux, hep-th/0010010.

[5] L.A. Pando Zayas and A.A. Tseytlin, 3-branes on a resolved conifold, hep-th/0010088.

[6] R. Gopakumar and C. Vafa, On the gauge theory/geometry correspondence, hepth/9811131.

[7] I.R. Klebanov and E. Witten, AdS/CFT correspondence and symmetry breaking, Nucl. Phys. B556 (1999) 89, hep-th/9905104.

[8] C. Vafa, Superstrings and topological strings at large $N$, hep-th/0008142.

[9] J. Polchinski and M. Strassler, The string dual of a confining four-dimensional gauge theory, hep-th/0003136. 
[10] J. M. Maldacena and C. Nuñez, Towards the large $n$ limit of pure $N=1$ super Yang Mills, hep-th/0008001.

[11] E. G. Gimon and J. Polchinski, Consistency Conditions for Orientifolds and DManifolds, Phys. Rev. D54 (1996) 1667, hep-th/9601038; M. R. Douglas, Enhanced gauge symmetry in M(atrix) theory, JHEP 9707 (1997) 004, hep-th/9612126.

[12] G.W. Gibbons and C.N. Pope, The positive action conjecture and asymptotically Euclidean metrics in quantum gravity, Commun. Math. Phys. 66 (1979) 267.

[13] D.N. Page, A physical picture of the K3 gravitational instanton, Phys. Lett. B80 (1978) 55.

[14] M. Berger, Bull. Soc. Math. France 83 (1955) 279.

[15] R.L. Bryant and S. Salamon, On the construction of some complete metrics with exceptional holonomy, Duke Math. J. 58 (1989) 829.

[16] G.W. Gibbons, D.N. Page and C.N. Pope, Einstein metrics on $S^{3}, \mathbb{R}^{3}$ and $\mathbb{R}^{4}$ bundles, Commun. Math. Phys. 127 (1990) 529.

[17] E. Bergshoeff, C.M. Hull and T. Ortin, Duality in the type II superstring effective action, Nucl. Phys. B451 (1995) 547, hep-th/9504081.

[18] P. Candelas and X.C. de la Ossa, Comments on conifolds, Nucl. Phys. B342 (1990) 246.

[19] R. Minasian and D. Tsimpis, On the geometry of non-trivially embedded branes, hepth/9911042.

[20] K. Ohta and T. Yokono, Deformation of conifold and intersecting branes, hepth/9912266.

[21] D.N. Page and C.N. Pope, Which compactifications of $D=11$ supergravity are stable?, Phys. Lett. B144 (1984) 346.

[22] A. Strominger, Heterotic solitons, Nucl. Phys. B343 (1990) 167, Erratum-ibid. B353 (1991) 565 .

[23] T. Eguchi and A.J. Hanson, Asymptotically flat self-dual solutions to Euclidean gravity, Phys. Lett. B74 (1978) 249. 
[24] M. Cvetič, S. Griffies and S. Rey, Static domain walls in N=1 supergravity, Nucl. Phys. B381 (1992) 301, hep-th/9201007.

[25] M. Cvetič and H. H. Soleng, Supergravity domain walls, Phys. Rept. 282 (1997) 159, hep-th/9604090.

[26] M. Cvetič, H. Lü and C. Pope, work in progress.

[27] M. Cvetič, S. S. Gubser, H. Lu and C. N. Pope, Symmetric potentials of gauged supergravities in diverse dimensions and Coulomb branch of gauge theories, Phys. Rev. D62 (2000) 086003, hep-th/9909121.

[28] S.W. Hawking, Gravitational instantons, Phys. Lett. A60 (1977) 81.

[29] G.W. Gibbons and S.W. Hawking, Gravitational multi-instantons, Phys. Lett. B78 (1978) 430 .

[30] M. Cvetič and C. Hull, Wrapped branes and supersymmetry, Nucl. Phys. B519 (1998) 141, hep-th/9709033.

[31] A. Sagnotti, A note on the Green-Schwarz mechanism in open-string theories, Phys. Lett. B294 (1992) 196.

[32] E. Lima, H. Lü, B.A. Ovrut and C.N. Pope, Instanton moduli and brane creation, Nucl. Phys. B569 (2000) 247, hep-th/9903001.

[33] M.J. Duff, H. Lü and C.N. Pope, Heterotic phase transitions and singularities of the gauge dyonic string, Phys. Lett. B378 (1996) 101, hep-th/9603037.

[34] N. Seiberg and E. Witten, Comments on string dynamics in six-dimensions, Nucl. Phys. B471 (1996) 121, hep-th/9603003.

[35] K. Behrndt, About a class of exact string backgrounds, Nucl. Phys. B455 (1995) 188, hep-th/9506106; R. Kallosh and A. Linde, Exact supersymmetric massive and massless white holes, Phys. Rev. D52 (1995) 7137, hep-th/9507022; M. Cvetič and D. Youm, Singular BPS saturated states and enhanced symmetries of four-dimensional $\mathrm{N}=4$ supersymmetric string vacua, Phys. Lett. B359 (1995) 87, hep-th/9507160.

[36] C.V. Johnson, A.W. Peet and J. Polchinski, Gauge theory and the excision of repulson singularities, Phys. Rev. D61 (2000) 086001, hep-th/9911161. 
[37] M.J. Duff, J.M. Evans, R.R. Khuri, J.X. Lu and R. Minasian, The octonionic membrane, Phys. Lett. B412 (1997) 281, Nucl. Phys. Proc. Suppl. 68 (1998) 295, hepth/9706124. 